\title{
PTEN regulates glioblastoma oncogenesis through chromatin-associated complexes of DAXX and histone H3.3
}

Jorge A. Benitez ${ }^{1}$, Jianhui Ma ${ }^{1}$, Matteo D'Antonio ${ }^{2,3}$, Antonia Boyer ${ }^{1}$, Maria Fernanda Camargo ${ }^{2,4}$, Ciro Zanca ${ }^{1}$, Stephen Kelly 5,6, Alireza Khodadadi-Jamayran"5,6, Nathan M. Jameson', Michael Andersen7, Hrvoje Miletic 7,8,9, Shahram Saberi ${ }^{10}$, Kelly A. Frazer $2,3,11$, Webster K. Cavenee ${ }^{1,2}$ \& Frank B. Furnari ${ }^{1,2,12}$

Glioblastoma (GBM) is the most lethal type of human brain cancer, where deletions and mutations in the tumour suppressor gene PTEN (phosphatase and tensin homolog) are frequent events and are associated with therapeutic resistance. Herein, we report a novel chromatin-associated function of PTEN in complex with the histone chaperone DAXX and the histone variant H3.3. We show that PTEN interacts with DAXX and, in turn PTEN directly regulates oncogene expression by modulating DAXX-H3.3 association on the chromatin, independently of PTEN enzymatic activity. Furthermore, DAXX inhibition specifically suppresses tumour growth and improves the survival of orthotopically engrafted mice implanted with human PTEN-deficient glioma samples, associated with global H3.3 genomic distribution changes leading to upregulation of tumour suppressor genes and downregulation of oncogenes. Moreover, DAXX expression anti-correlates with PTEN expression in GBM patient samples. Since loss of chromosome 10 and PTEN are common events in cancer, this synthetic growth defect mediated by DAXX suppression represents a therapeutic opportunity to inhibit tumorigenesis specifically in the context of PTEN deletion.

\footnotetext{
${ }^{1}$ Ludwig Institute for Cancer Research, La Jolla, California 92093-0660, USA. ${ }^{2}$ The Moores Cancer Center, University of California San Diego, La Jolla, California 92093, USA. ${ }^{3}$ Department of Pediatrics and Rady Children's Hospital, University of California San Diego, La Jolla, California 92093, USA. ${ }^{4}$ Sanford Consortium for Regenerative Medicine, University of California, San Diego, 3855 Health Science Drive, La Jolla, California 92037, USA. ${ }^{5}$ Department of Pathology, Laura \& Isaac Perlmutter Cancer Center, and The Helen L. and Martin S. Kimmel Center for Stem Cell Biology, NYU School of Medicine, New York, New York 10016, USA. ${ }^{6}$ Center for Health Informatics and Bioinformatics, NYU School of Medicine, New York, New York 10016, USA.

7 Department of Pathology, Haukeland University Hospital, 5021 Bergen, Norway. ${ }^{8}$ Department of Biomedicine, University of Bergen, 5009 Bergen, Norway. ${ }^{9} \mathrm{KG}$ Jebsen Brain Tumour Research Center, University of Bergen, 5009 Bergen, Norway. ${ }^{10}$ Department of Neurosciences, University of California, San Diego, La Jolla, California 92093, USA. ${ }^{11}$ Institute for Genomic Medicine, University of California San Diego, La Jolla, California 92093, USA. ${ }^{12}$ Department of Pathology, University of California San Diego, La Jolla, California 92093, USA. Correspondence and requests for materials should be addressed to F.B.F. (email: ffurnari@ucsd.edu).
} 
G lioblastoma (GBM) is the most common and aggressive form of cancer of the central nervous system. The TCGA (The Cancer Genome Atlas) data indicate that about 50\% of GBMs harbour somatic alterations in the phosphatidylinositol $3-\mathrm{OH}$ kinase pathway ${ }^{1,2}$. One of the essential regulators of this pathway that is significantly altered in GBMs $(30-40 \%)$ is the PTEN tumour suppressor gene ${ }^{1,3}$, which encodes a phosphatase responsible for the removal of phosphate from the $3^{\prime}$ position of the phospholipid second messenger phosphatidylinositol-3,4,5trisphosphate, thus opposing mitogenic signalling mediated by class 1 phosphatidylinositol 3-OH kinases ${ }^{4}$. The loss of PTEN function has been mechanistically linked to metastasis ${ }^{5}$, and lack of radio-therapy ${ }^{6}$ and chemo-therapy $y^{7,8}$ response in brain and breast cancer patients, indicating that PTEN is a key regulator of tumour sensitivity to multiple therapeutic approaches. It has been well established that different epigenetic, transcriptional and post-translational mechanisms control the level and function of PTEN. Moreover, PTEN protein-protein interactions can also affect its tumour suppressor properties ${ }^{9-11}$.

GBMs undergo genetic lesions that affect the epigenomic machinery that controls histone modifications, DNA methylation and gene expression. One such target is the histone $\mathrm{H} 3$ variant, H3.3, which is incorporated into chromatin in a cell cycle independent manner and is associated with transcriptionally active and silent chromatin in somatic and embryonic cells ${ }^{12,13}$. Two distinct and mutually exclusive $\mathrm{H} 3.3$ mutations (K27M and G34R/V) have been identified in paediatric $\mathrm{GBMs}^{14,15}$, associated with global downregulation of the repressive histone mark H3K27me3, DNA hypomethylation ${ }^{16-18}$, ALT (Alternative Lengthening of Telomere) phenotyp $\mathrm{e}^{14}$ and upregulation of the MYCN pathway ${ }^{19}$. In adult GBMs, H3.3 expression is repressed by MLL5 (Mixed Lineage Leukemia 5), leading to chromatin reorganization and self-renewal ${ }^{20}$. Histone chaperones that are involved in the recruitment of H3.3 to chromatin are DAXX (death-domain associated protein), ATRX (alpha-thalassaemia/ mental retardation X-linked syndrome protein) and HIRA (histone cell cycle regulator) ${ }^{12,21-24}$. Somatic mutations of DAXX and ATRX have been reported in adult pancreatic neuroendocrine tumours ${ }^{25}$, low grade gliomas ${ }^{26,27}$ and paediatric high grade gliomas $^{14}$; however, genetic alterations of DAXX are rare in other types of cancers.

To date, various approaches have been used to target chromatin deregulation in cancer cells ${ }^{28-30}$. Here, we report a novel chromatin-associated function of the PTEN tumour suppressor that represses oncogene expression and tumour growth in patientderived glioma xenografts through DAXX-H3.3 association. We show that DAXX physically interacts with PTEN, and PTEN regulates H3.3 loading on chromatin by limiting DAXX interactions with this histone, and thereby controls expression of several tumour-promoting genes. Moreover, DAXX inhibition affects global H3.3 deposition and gene expression, specifically suppresses intracranial tumour growth and significantly improves the survival of PTEN-null glioma-bearing mice. These results demonstrate a synthetic growth defect that occurs due to loss of these two tumour suppressor genes.

\section{Results}

PTEN interacts with DAXX and controls oncogene expression. Several reports have shown that PTEN can control tumorigenesis independent of its enzymatic activity, through its interaction with specific nuclear proteins ${ }^{9-11}$. To uncover further PTEN-nuclear interactions, an in silico analysis was performed using the Human Interactome Map (HiMAP) $)^{31}$ bioinformatics site. From this analysis four interactome complexes were predicted for PTEN and for DAXX, that included previously reported interactions
PTEN-TP53 (ref. 10), -MCRS1 (ref. 11) and -PML ${ }^{9}$ and DAXXTP53 (ref. 32), -MCRS1 (ref. 33) and -PML ${ }^{34}$, and a new interaction consisting of PTEN-DAXX (Fig. 1a). Since DAXX is a key regulator of gene expression 35,36 and has been shown to indirectly regulate PTEN stability ${ }^{37}$, we determined whether PTEN and DAXX could be physically associated. Pulldown assays using purified recombinant proteins (Fig. 1b) and total protein lysates from glioma cells that expressed different endogenous PTEN and DAXX levels (Fig. 1c) demonstrated that PTEN can physically interact with DAXX. To map the interaction domains for each protein, various GFP-PTEN and Flag-DAXX deletion constructs were co-transfected with full length Flag-DAXX or GFP-PTEN, respectively. These experiments showed that DAXX bound to the unfolded PTEN-hinge domain (amino acids 186-202) (Fig. 1d), while PTEN bound DAXX through its histone-binding domain (Fig. 1e).

DAXX is a histone chaperone protein that directly interacts with the histone H3.3 variant and facilitates its deposition on chromatin $23,24,38$. Using protein lysates from patient-derived glioma neurospheres (GBM-PDX) that express endogenous PTEN, DAXX and H3.3, we examined whether the PTEN-DAXX complex identified above interacts with H3.3. As shown in Fig. 2a and Supplementary Fig. 1a,b, endogenous PTEN co-immunoprecipitated with H3.3 and DAXX in GBM-sphere samples. We corroborated this result by performing sequential immunoprecipitation from nuclear extracts of a PTEN-null glioma-sphere that over-expresses exogenous PTEN (HK281-PTEN) (Fig. 2b), and also by quantitative immunofluorescence of endogenous proteins in GBM-spheres (Fig. $2 \mathrm{c}$ and Supplementary Fig. 1c,d Pearson's coefficient in co-localized region equal to 0.4168 for TS576, 0.4526 for GBM39, 0.7665 for TS543 and 0.7344 for TS528 GBM-spheres). These results confirm the presence of a nuclear PTEN-DAXX-H3.3 tripartite complex in patient-derived GBM neurospheres.

We then asked if the PTEN-DAXX complex modulated the histone H3.3 chaperone function of DAXX. By immunonprecipitating DAXX from nuclear protein extracts of Pten-wt and Pten-null MEF (mouse embryonic fibroblast) cells we observed that the levels of H3.3 that co-immunoprecipitated with DAXX increased twofold in Pten-null cells when compared with Pten-wt cells (Fig. 2d, left panel and Supplementary Fig. 1e). Conversely, upon reconstitution of PTEN expression in a PTEN-deficient glioma cell line (U87), we observed a threefold reduction in the levels of H3.3 bound to DAXX (Fig. 2d, right panels and Supplementary Fig. 1e). These results indicate that DAXX-H3.3 interaction could be regulated by PTEN expression.

H3.3 deposition has been associated with both active and repressive chromatin ${ }^{12,13}$, while DAXX has been associated exclusively with gene repression ${ }^{35,36}$. This dichotomy led us to investigate the role of PTEN in the regulation of $\mathrm{H} 3.3$ deposition on chromatin and consequent gene expression. Four genes, either involved in neural stem cell proliferation and/or neuronal activity that have been reported to be regulated by $\mathrm{PTEN}^{39-43}$ and $\mathrm{DAXX}^{35,38}$, were analysed (CCND1, MYC, FOS and BCL2). Both RT-qPCR and anti-H3.3 chromatin immunoprecipitation (ChIP) were performed in PTEN-NULL and PTEN-WT MEFs, glioma cells and GBM-spheres. These experiments showed an inverse correlation between gene expression (Fig. 2e,f and Supplementary Fig. 2a,b) and H3.3 enrichment (Fig. 2g,h and Supplementary Fig. 2c) in PTEN-deficient cells compared with PTEN-WT cells $(P<0.0001)$, suggesting that PTEN represses oncogene expression by recruiting H3.3 to chromatin. These data were corroborated by performing ChIP assays for other markers of active (RNA pol-II) and repressive (H3K27me3) chromatin, which showed a strong correlation between high H3.3 levels and a high H3K27me3 signal in the presence of PTEN expression (Supplementary Fig. 2d, 
a
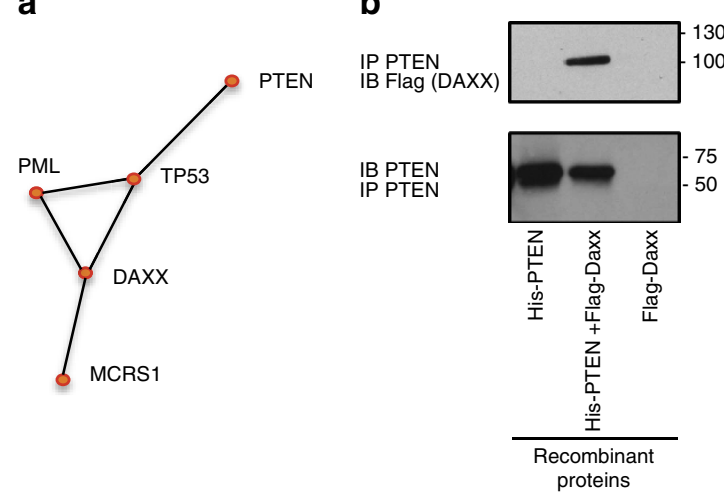

C

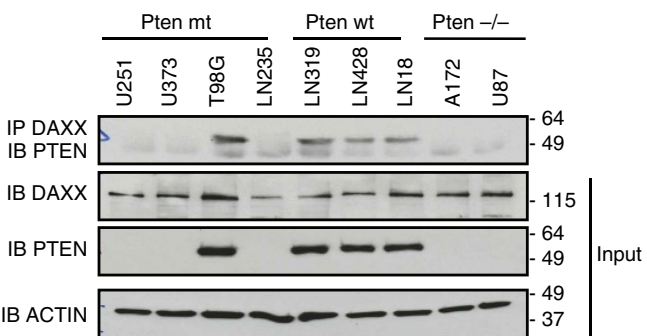

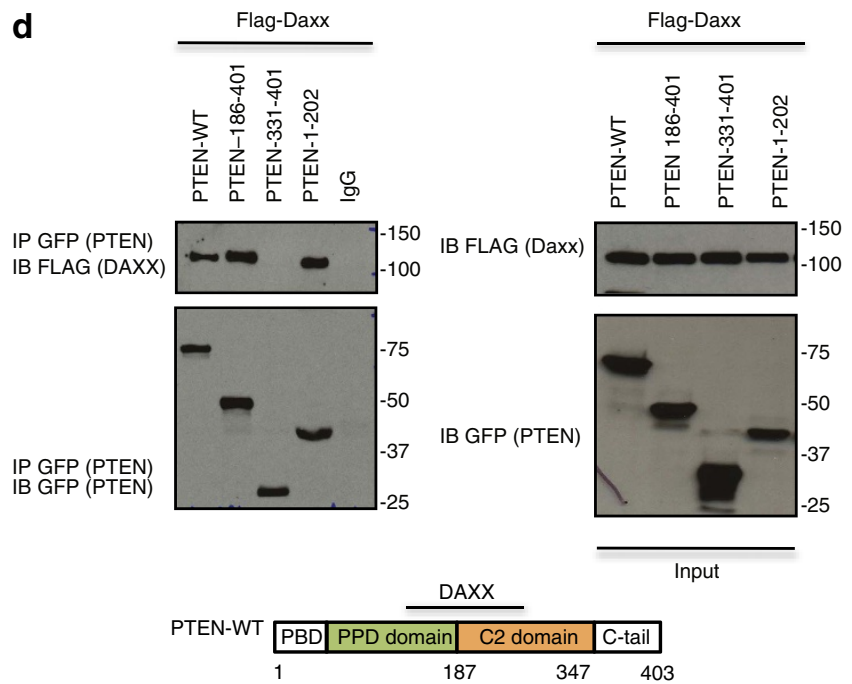

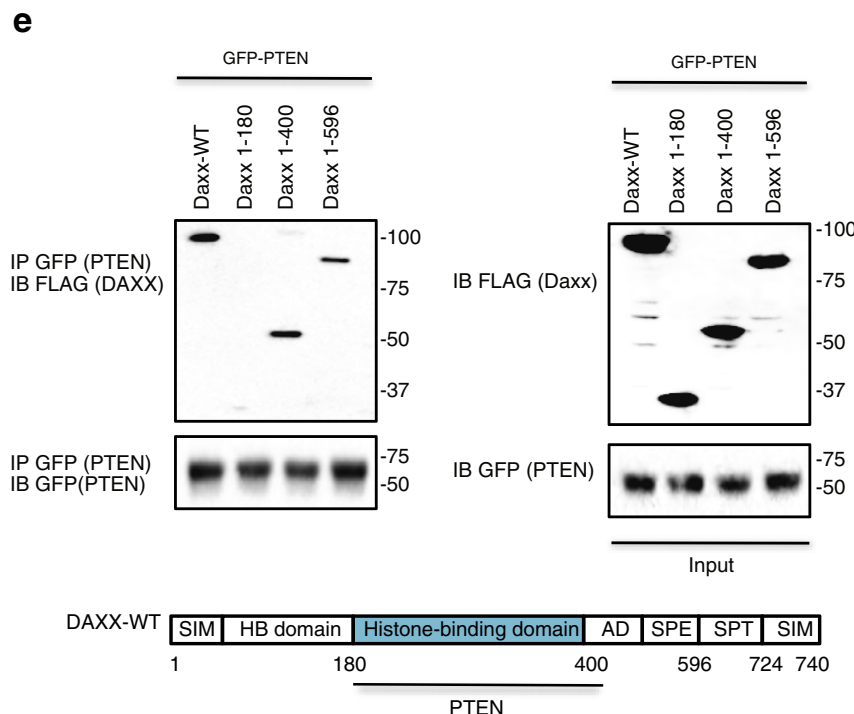

Figure 1 | DAXX interacts with PTEN. (a) Predicted protein-protein interactions between PTEN, TP53, PML, DAXX and MCRS1 using the human protein interaction map. (b) In vitro pull-down using mixed Flag-DAXX and His-PTEN recombinant proteins immunoprecipitated (IP) with anti-PTEN and immunoblotted (IB) with anti-Flag. (c) Co-immunoprecipitation assay using whole cell protein lysates from different cell lines expressing endogenous DAXX and PTEN. IP anti-DAXX and IB anti-PTEN. (d,e) PTEN and DAXX interacting domains were mapped using 293 T cells co-transfected with Flag-Daxx-wt or GFP-PTEN and deletion constructs of each protein.

$P<0.0001)$; and an inverse correlation between low $\mathrm{H} 3.3$ levels and a high RNA Pol-II signal in PTEN-deficient cells (Supplementary Fig. 2e, $P<0.001)$. Taken together, these data show that PTEN represses gene expression by increasing the levels of $\mathrm{H} 3.3$ bound to chromatin.

Regulation of gene expression by PTEN has been reported previously ${ }^{9}$. To determine if changes in the amount of H3.3 bound to chromatin were dependent on the lipid phosphatase activity of PTEN, ChIP assays were performed with anti-H3.3 in PTEN-deficient cells reconstituted with PTEN-wild type (PTEN-WT) or a PTEN-lipid and protein phosphatase inactive mutant (PTEN-G129R). As shown in Supplementary Fig. 2f,g, PTEN-G129R increased the amount of H3.3 bound to chromatin to the same level as PTEN-WT and also reduced the levels of H3.3 bound to DAXX (Supplementary Fig. 2h), indicating that H3.3 deposition on chromatin was regulated by PTEN independently of its phosphatase activity.

DAXX has been shown to compete with DNA for H3.3-H4 tetramer formation ${ }^{44,45}$ and we found that PTEN competes with H3.3 for the DAXX-histone binding domain (Fig. 1e). Based on these results, the effect of PTEN on DAXX cellular compartmentalization was investigated using cell fractionation. As shown in Fig. 2i, a reduction in the DAXX-chromatin fraction and a reciprocal increase in the DAXX soluble nuclear fraction occurred in Pten-null cells compared with Pten-wt cells. Upon PTEN reconstitution in PTEN-deficient cells, the amount of DAXX associated with chromatin increased from 37 to $61 \%$ (Supplementary Fig. 3a). Corroborating these results, DAXX immunofluorescence quantification in PTEN-WT cells displayed a specific signal associated with nuclear bodies (Fig. $2 \mathrm{j}-1$ and Supplementary Fig. 3b); however, the DAXX signal was highly diffuse in the nucleus and cytosol in PTEN-deficient cells (Fig. 2j-l and Supplementary Fig. 3b). No changes in nuclear body distribution of ATRX and HIRA were observed in Pten-wt and Pten-null cells (Supplementary Fig. 3c,d). In order to interrogate if ATRX or an ATRX mutant $\left(\mathrm{R}_{\left.1426^{*}\right)}\right.$ that is present in gliomas affect DAXXPTEN and/or DAXX-H3.3 association we overexpressed these constructs and analysed their effect. No changes in DAXX-PTEN and DAXX-H3.3 associations were observed after overexpressing ATRX-WT or ATRX-R1426* mutant (Supplementary Fig. 3e). Taken together, these results show that PTEN regulates oncogene expression by controlling DAXX-H3.3 association and deposition on chromatin independently of ATRX.

DAXX suppression compromises PTEN-deficient cells. To investigate if $D A X X$ disruption can restore $\mathrm{H} 3.3$ deposition to the oncogene promoters interrogated in Fig. 2, and consequently 
a

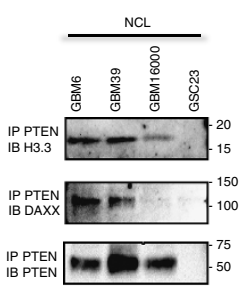

b

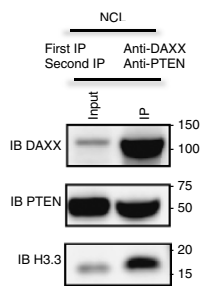

C

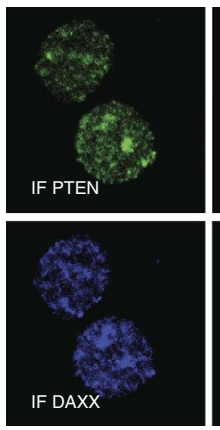

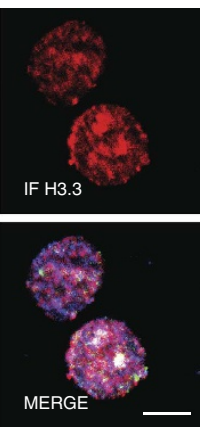

f

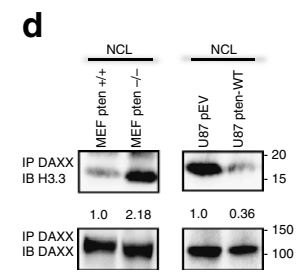

e

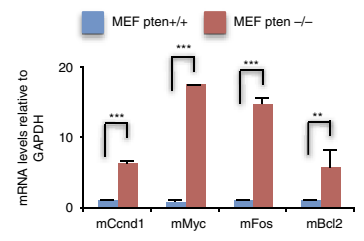

g
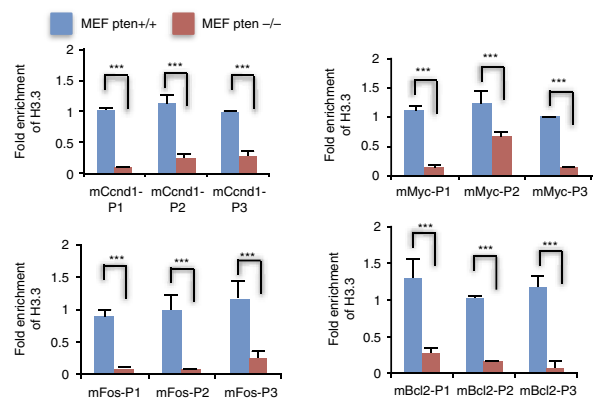

h

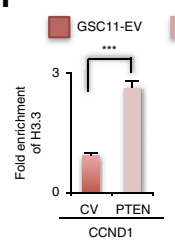

GSC11-PTEN
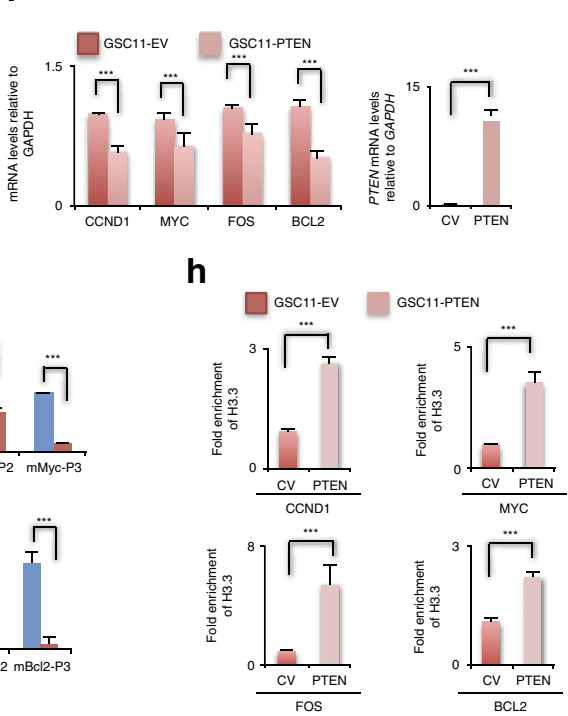

i

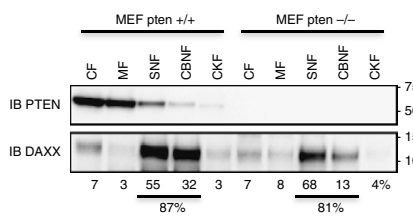

I

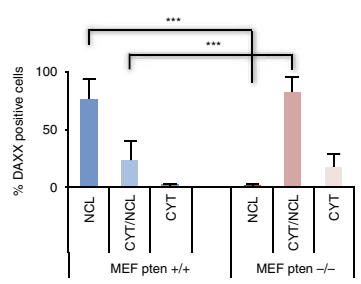

j

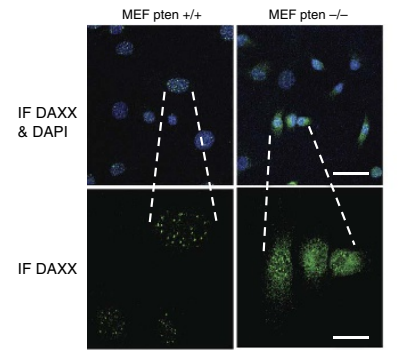

k

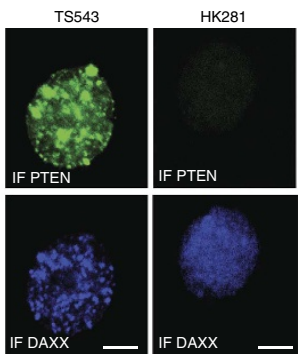

Figure 2 | PTEN represses oncogene expression by controlling H3.3 deposition on chromatin and H3.3-DAXX interaction. (a) Co-immunoprecipitation analysis of anti-PTEN followed by anti-H3.3 or anti-DAXX immunoblotting were performed using nuclear protein (NCL) from patient-derived glioma spheres. (b) Sequential immunoprecipitation from nuclear extracts of HK281-PTEN GBM-spheres. First IP anti-DAXX and second IP anti-PTEN, and immunoblots anti-DAXX, anti-PTEN and anti-H3.3. (c) Confocal immunofluorescence analysis of endogenous PTEN, H3.3 and DAXX proteins in TS576 GBM-spheres (Pearson's coefficient in colocalized region equal to 0.4168). Scale bar, $5 \mu \mathrm{m}$. (d) Nuclear proteins from MEF pten $+/+$ and MEF pten $-/-$ (left), and U87 glioma cells (right) transfected with empty vector pEV or PTEN-WT were immunoprecipitated with anti-DAXX followed by immunoblotting with anti-H3.3. (e,f) mRNA expression analysis of CCND1, MYC, FOS and BCL2 by RT-qPCR in PTEN-WT or PTEN-deficient cells ( $n=3$ biological samples with three replicates each, ${ }^{\star \star} P<0.001,{ }^{\star \star \star} P<0.0001$, one-way ANOVA). (g,h) ChIP-qPCR of H3.3 in PTEN-WT or PTEN-deficient cell, using different sets of primers that anneal within 1-2 Kb of the transcription start site. For ChIP assays, bar graphs indicate fold enrichment of $\mathrm{H} 3.3$ over input $(n=3$ biological samples with three replicates each, ${ }^{\star \star \star} P<0.0001$, one-way ANOVA). (i) Cellular fractionation was carried out in MEF cells followed by anti-DAXX and anti-PTEN immunoblotting. DAXX expression was quantified by densitometry analysis. (j,k) Representative immunofluorescence images of endogenous DAXX expression in MEFs (j) or GBM-spheres (k). For $\mathbf{j}$ top scale bar is $20 \mu \mathrm{m}$ and bottom scale bar is $50 \mu \mathrm{m}$. For $\mathbf{k}$ scale bar is $5 \mu \mathrm{m}$. (I) Percentage of DAXX-positive cells in different cellular compartments in Pten-wt and Pten-null MEFs. NCL: nuclear; CYT/NCL: cytosolic and nuclear; CYT: cytosolic; NS: no significant differences. Error bars represent s.e.m. from three different experiments, ${ }^{\star \star \star} P<0.0001$, one-way ANOVA. 
suppresses their expression in PTEN-deficient cells, lentiviralencoded shRNAs targeting DAXX were used to generate stable DAXX-knockdown (Daxx-kd) in glioma cell lines and GBM-PDX neurospheres. Upon immunoblot analysis, DAXX-kd cells showed a decrease in CYCLIN-D1, MYC, FOS and BCL2 protein expression when compared to shControl cells (Fig. 3a and Supplementary Figs $4 \mathrm{a}$ and $5 \mathrm{a}$ ). In concordance with these results, H3.3 enrichment on the promoters of CCND1, MYC, FOS and $B C L 2$ was observed after DAXX knockdown in PTEN-deficient cells $(P<0.001)$ compared with shControl cells (Fig. $3 c$ and Supplementary Figs $4 \mathrm{~b}$ and $5 \mathrm{~b}$ ). By performing a promoter reporter assay we corroborated a direct repression of CYCLIND1 and MYC promoter activity in DAXX-kd/PTEN-null cells compared with shControl cells (Supplementary Fig. 4c), thus confirming direct DAXX-mediated regulation of these genes in PTEN-deficient cells.

Because MYC and CYCLIN-D1 play important roles in regulating cell cycle progression and proliferation ${ }^{46,47}$, the effect of DAXX inhibition on these cellular functions was analysed in PTEN-deficient cells. These experiments showed that a greater percentage of cells were arrested in the G1 and G2 phases of the cell cycle in DAXX-kd/PTEN-null cells $(15$ and $20 \%, P<0.001)$ compared to shControl cells (Supplementary Figs $4 \mathrm{~d}$ and $5 \mathrm{c}$ ). Knocking down DAXX expression in PTEN-deficient cells also suppressed cell proliferation $(P<0.001)$, while shControl/PTENnull cells proliferated 2.5-fold faster than DAXX-kd/PTEN-null cells (Supplementary Figs 4e and 5d). No changes in cell cycle progression were seen in any of the GBM-spheres cells after $D A X X-k d$ (Supplementary Fig. 5e), explained in part by their quiescent or slowly proliferative nature ${ }^{48,49}$. Together, these results illustrate that DAXX inhibition in PTEN-deficient cells restores the deposition of $\mathrm{H} 3.3$ on chromatin, promotes oncogene repression and compromises cellular proliferation.

In order to confirm that DAXX-knockdown directly affects oncogene expression through $\mathrm{H} 3.3$ and to eliminate the possibility of off-target effects, we reconstituted DAXX-kd/ PTEN-deficient cells with a DAXX-shRNA-resistant construct (HA-DAXX). As shown in Supplementary Fig. 6a,b protein and mRNA levels of CCND1, MYC, FOS and BCL2 genes were restored after DAXX re-expression in DAXX-kd cells. H3.3 enrichment on the promoter of those genes was also restored to normal levels compared with shControl cells after reconstitution of HA-DAXX or PTEN re-expression in DAXX-kd/PTENdeficient cells (Fig. 3e,f and Supplementary Fig. 6c), as well as cellular proliferation (Supplementary Fig. 6d) and oncogene promoter activity (Supplementary Fig. 6e,f); confirming that DAXX was responsible for these cellular functions.

To test if these cellular and molecular effects observed after $D A X X$ knockdown were PTEN dependent, DAXX expression was knocked down in glioma cell lines and GBM-spheres that express wild-type PTEN. No significant changes in total protein levels of CYCLIN-D1, MYC, FOS and BCL2 were detected after DAXX knockdown in PTEN-WT cells compared with shControl/PTENWT cells (Fig. 3b and Supplementary Fig. 7a,e). Additionally, no or faint changes in the enrichment of $\mathrm{H} 3.3$ (Fig. 3d and Supplementary Fig. 7b) or in cell cycle progression (Supplementary Fig. 7c,f) were observed in Daxx-kd/PTEN-WT cells in comparison with shControl cells. DAXX knockdown in PTEN$W T$ cells slightly affected cell proliferation in glioma cells (Supplementary Fig. 7d,g), which is perhaps related to other molecular mechanisms associated with $\mathrm{DAXX}^{50}$. These data indicate that PTEN is a critical component for H3.3 chromatin deposition-associated gene repression, while DAXX suppression, in the context of PTEN deficiency, re-establishes H3.3-mediated oncogene repression (Fig. 6h and Supplementary Fig. 21).
Similarly, we observed a decrease in H3.3 loading on oncogene promoters (Supplementary Fig. 8a) and an increase in oncogene expression (Supplementary Fig. 8b) in Daxx-null MEF cells (MEF Daxx -/-) compared with Daxx-expressing cells (MEF Daxx $+/+$ ). Furthermore, Pten inhibition in Daxxdeficient cells (MEF Daxx $-/-$ shPten, Supplementary Fig. 8c) restored H3.3 enrichment on the chromatin (Supplementary Fig. 8d) and repressed oncogene expression (Supplementary Fig. 8e), blocking cell proliferation of Daxx-deficient cells (Supplementary Fig. 8f,g). We speculate that oncogene expression is driven in Daxx-deficient MEFs in part because Pten still binds to H3.3 in the absence of Daxx expression (Supplementary Fig. $8 \mathrm{~h})$.

$D A X X$ inhibition affects PTEN-null glioma spheres. To further identify genome-wide H3.3 distribution changes after DAXX inhibition in PTEN-null/GBM-PDX samples, we performed unbiased chromatin immunoprecipitation sequencing (ChIP-seq) (Supplementary Fig. 9a). We observed that most of the H3.3 enrichment in DAXX knockdown cells occurred in distal intergenic $(37 \%)$ and intronic $(36 \%)$ regions and about $10 \%$ in promoter regions (Fig. 4a). Of the 3,200 peaks displaying a H3.3 differential binding pattern (DiffBind), there were 1,425 genes with a positive fold change (FC) and 326 genes with a negative FC $(P<0.05 \& \log 2$ (Fold Change) $>1$, Fig. 4b). Gene ontology and pathway analysis of H3.3 differentially enriched genes correlated with pathways associated with metabolic process, nervous system development, neuronal differentiation and the cell cycle (Fig. 4c and Supplementary Fig. 9b,c). Furthermore, to examine transcriptome changes in DAXX-kd/PTEN-null GBM cells we performed RNA sequencing (RNA-seq) (Supplementary Fig. 11a). Of the 1,403 genes showing differential expression (DiffExp) between cells treated with shControl and cells treated with shDAXX, there were 846 upregulated genes and 557 downregulated genes $(P<0.05$ and $\log 2$ (FoldChange) $>1$, Fig. $4 \mathrm{~d}$ ). $D A X X$-kd resulted in a significant upregulation of several tumour suppressor genes and downregulation of various oncogenes, including CCND1, MYC, FOS, SOX2 and OLIG2 (Fig. 4e and Supplementary Fig. 10). Additionally, the most significant pathways were neuronal differentiation, nervous system development, neurogenesis and regulation of cell differentiation (Supplementary Fig. 11b).

Further analyses were performed overlapping the H3.3 DiffBind peaks from ChIP-seq with DiffExp genes from RNA-seq. In total 1,390 genes were overlapping between the two data sets, where a subset of 133 genes were upregulated and enriched for $\mathrm{H} 3.3$, and 37 genes were downregulated and enriched for H3.3 $(P<0.05$ and log2foldChange $>1)$ (Fig. 4f). Gene ontology analysis confirmed enrichment in pathways associated with nervous system development, regulation of MAPK cascade and neuronal differentiation (Supplementary Fig. 11c,d). These data show that DAXX inhibition robustly alters H3.3 genomic distribution leading to affects on gene expression in PTEN-null/GBM neurospheres.

We next studied whether DAXX inhibition can compromise the oncogenic behaviour of GBM-PDX cells grown in neurosphere conditions (Supplementary Fig. 12a). Immunoblot analysis of transcription factors involved in the development of gliomas

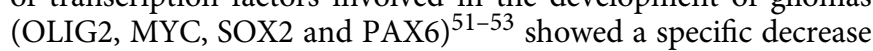
in expression in PTEN-deficient cells (GSC11, GSC23, HK281) compared with PTEN-WT cells (GBM6, TS675, GBM39, TS543) (Supplementary Fig. 12b,c), suggesting that DAXX disruption in PTEN-null/GBM-PDXs affects the expression of transcription factors implicated in gliomagenesis. A downregulation of ATRX expression was also observed after DAXX inhibition in both 
a

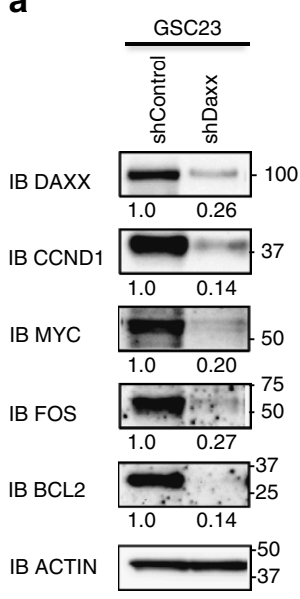

c
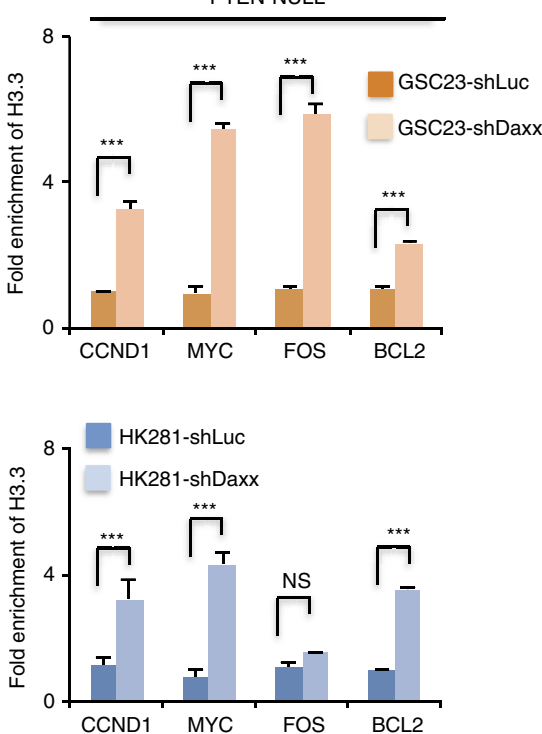

e

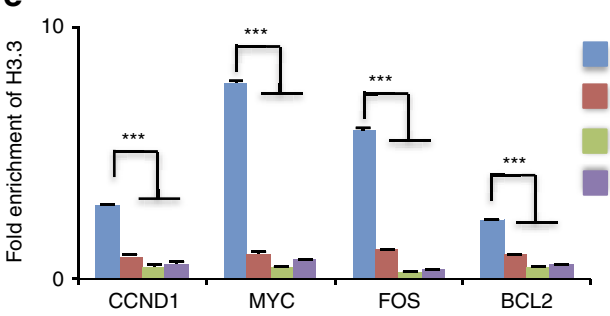

b

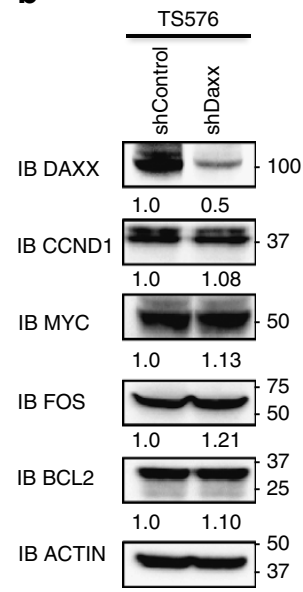

d
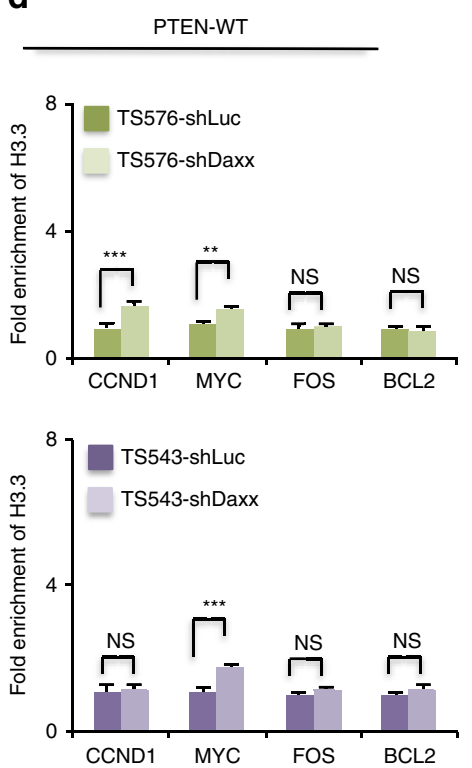

f
GSC23-shDaxx

GSC23-shLuc

GSC23-shDaxx\&HADAXX

GSC23-shDaxx\&PTEN

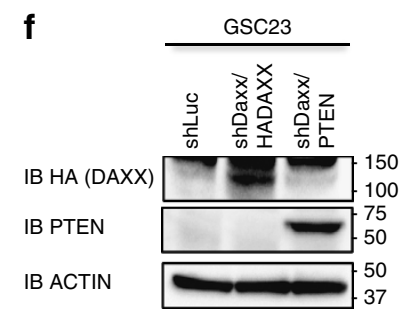

Figure 3 | DAXX disruption compromises gene expression and restores H3.3 deposition in PTEN-deficient cells. (a,b) Total proteins were analysed by western blot in PTEN-deficient (a) or PTEN-expressing (b) GBM-spheres transduced with lentivirus shRNA control (shLuc) or shRNAs targeting DAXX (shDaxx). Actin is a loading control. (c,d) anti-H3.3 ChIP-qPCR was performed in PTEN-deficient (c) or PTEN-WT (d) GBM-spheres with stable knockdown of DAXX or with shControl. (e) ChIP-qPCR of H3.3 in Daxx-kd/PTEN-deficient GBM-spheres expressing a HA-DAXX-shRNA-resistant vector or PTEN-WT. For ChIP assays, bar graphs indicate fold enrichment of $\mathrm{H} 3.3$ over input $\left(n=3\right.$ biological samples with three replicates each, ${ }^{\star \star} P<0.001,{ }^{\star \star \star} P<0.0001$, one-way ANOVA). (f) Immunoblot analysis of HA-DAXX and PTEN expression in DAXX-kd or shControl GSC23 neurospheres. NS: no significant differences. Error bars represent s.e.m. from three different experiments. Numbers under the blots indicate fold ratios of protein levels relative to shControl after normalization to actin.

conditions (Supplementary Fig. 12c), PTEN-expressing and PTEN-null GBM-spheres, as has previously been reported by other groups ${ }^{12,23}$.

Additionally, we determined if $D A X X$ knockdown affects GBM-PDX proliferation. Knocking down DAXX resulted in a specific reduction of number and size of spheres formed by
PTEN-null (GSC11 and GSC23) cells, compared with PTENexpressing (GBM6 and TS576) cells (Fig. 4g,h), $P<0.001$. Furthermore, using an in vitro limiting dilution assay, DAXX knockdown resulted in a three- to eightfold reduction in the self-renewal capacity of PTEN-null GBM-PDXs compared with DAXX-kd/PTEN-WT spheres (Fig. 4i, Supplementary Fig. 13). 
In order to investigate if DNA replication was affected after DAXX disruption, since it has been reported that PTEN regulates DNA regulation and repair ${ }^{9,54}$, we quantified the percentage of positive cells relative to two components of the fork replication complex (RPA32-P and ATRIP-P). As is shown in
Supplementary Fig. 14 DAXX inhibition increases the percentage of RPA32-P- and ATRIP-P-positive cells (GSC23, $17 \%$ and $12 \%$ increase, respectively) specifically in PTEN-null GBM-spheres. We next evaluated the effect of DAXX suppression on the differentiation capacity of GBM-PDX neurospheres.
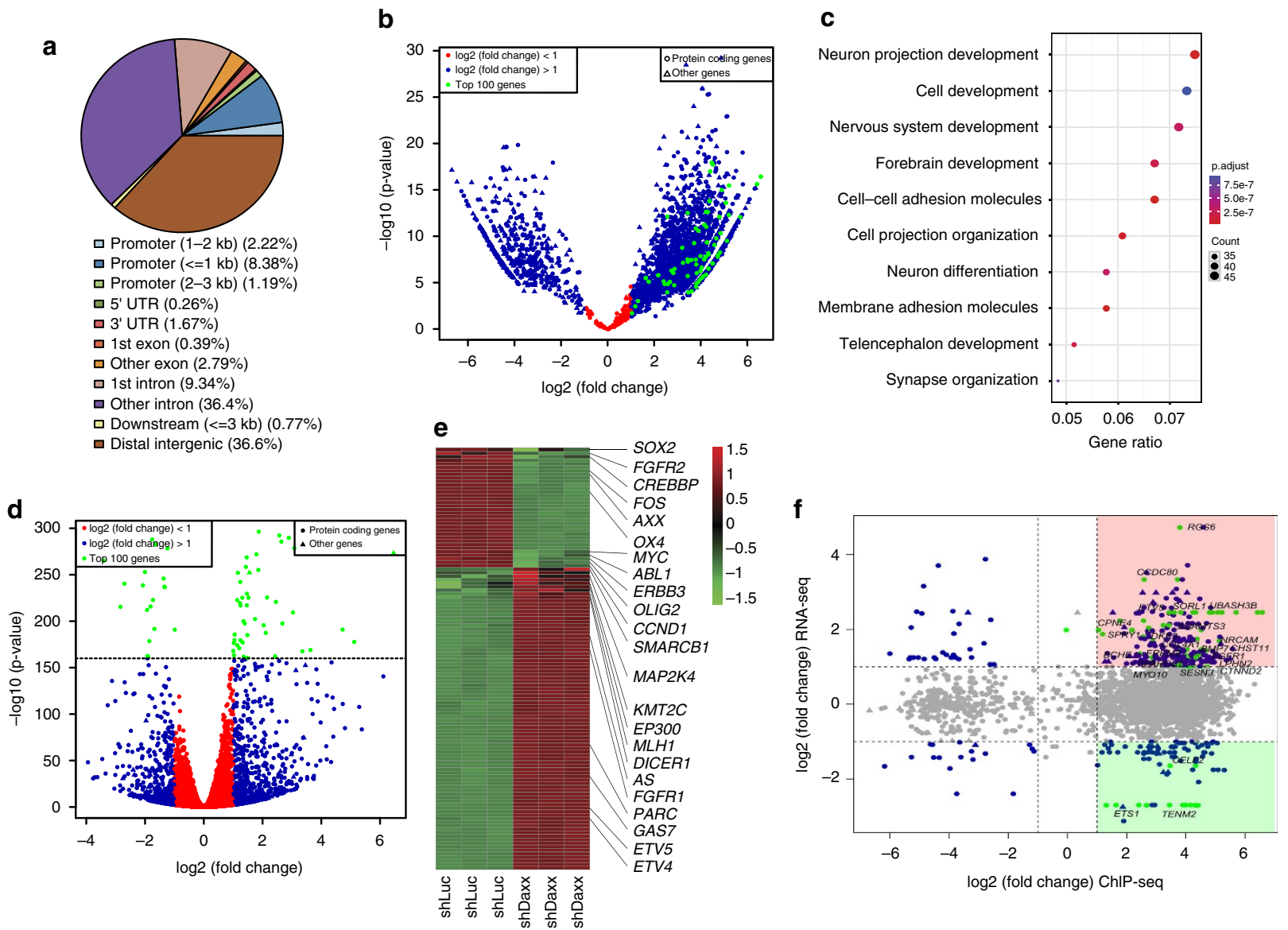

g
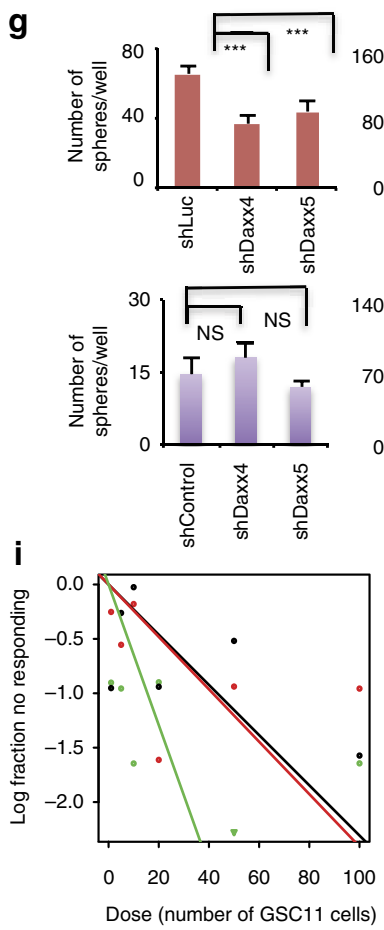
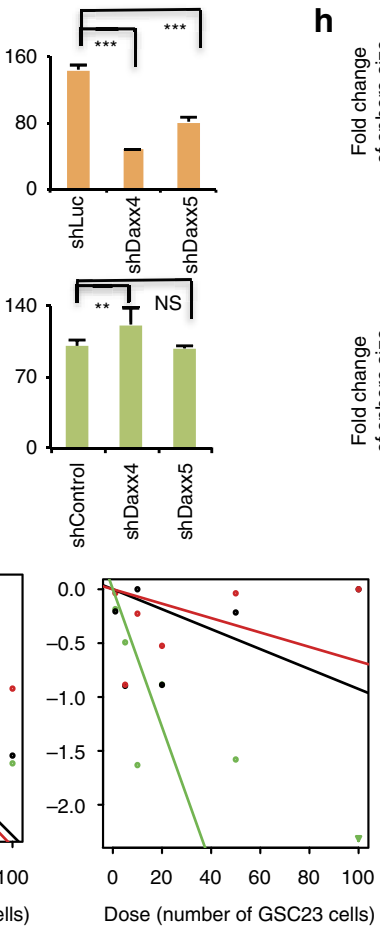

h
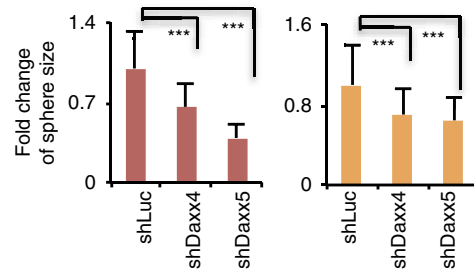

GSC11

GSC23

GBM6
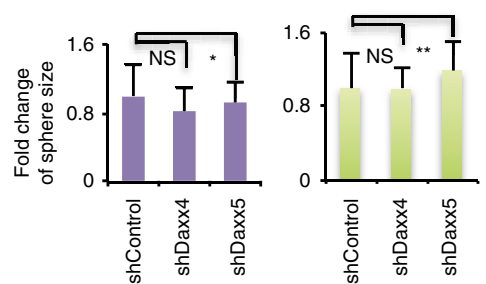
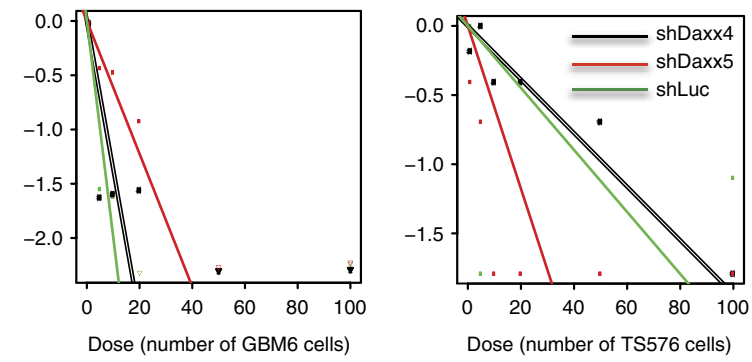
In contrast to shControl cells, there was a $30-50 \%$ decrease in OLIG2 (Supplementary Fig. 15a, $P<0.0010 .0001$ ) and a $20-30 \%$ decrease in GFAP-positive cells (Supplementary Fig. 15b, $P<0.001)$ when DAXX knockdown cells were incubated in differentiation conditions. In general, these results illustrate that $D A X X$ inhibition, independent of ATRX, disrupts GBM-PDX oncogenic properties selectively in a PTEN-deficient genetic background, in part through downregulation of transcription factors that preserve glioma proliferation and upregulation of tumour suppressor genes.

$D A X X$ inhibition suppresses tumor growth and improves survival. To assess whether $D A X X$-knockdown affects intracranial tumour growth, we infected glioma-PDX neurospheres with a lentivirus-encoded shRNA targeting DAXX (shDaxx) or control shRNA (shLuc), along with a lentivirus expressing a near-infrared fluorescent protein. Imaging using fluorescence molecular tomography showed a decrease in fluorescence signal in mice engrafted with shDAXX/PTEN-deficient (GSC11, GSC23, HK281) GBM neurospheres in comparison with animals implanted with shDAXX/PTEN-WT (TS576, GBM39, GBM6, TS543) GBM cells (Fig. 5a,b Supplementary Fig. 16c,d). Relative fluorescence quantification showed a statistically significant $(P<0.0001)$ reduction in tumour growth for DAXX-kd/PTENdeficient glioma xenografts (Fig. 5c, Supplementary Fig. 16a,e) compared with mice implanted with DAXX-kd/PTEN-WT GBM spheres (Fig. 5e, Supplementary Fig. 16b,e). Moreover, survival analysis showed mice intracranially engrafted with DAXX-kd/ PTEN-deficient GBM cells (Fig. 5d, Supplementary Fig. 16f) survived significantly $(P<0.0001)$ longer than animals implanted with DAXX-kd/PTEN-WT GBM spheres (Fig. $5 \mathrm{f}$ and Supplementary Fig. 16f).

We validated that $D A X X$ inhibition directly affects tumour size and survival of PTEN-null glioma xenografts by restoring DAXX or PTEN expression in DAXX-kd/PTEN-deficient engrafted mice. Neither changes in fluorescence signal nor significant differences in tumour size and survival were observed in DAXX-kd/PTEN-null xenografts (Fig. 5g-i, Supplementary Fig. 16g) after re-expression of HA-DAXX or PTEN-WT (Fig. 5j). Histological analyses of mouse brain tumours showed angiogenesis, haemorrhagic areas, necrosis and high mitotic activity in tumours derived from shLuc and shDAXX/PTEN-WT GBM-spheres (TS576; Supplementary Fig 17a,b,g). In contrast, no haemorrhagic areas, angiogenesis, or necrosis, and low mitosis were observed in brains implanted with shDAXX/PTEN-null GBM-spheres compared to shLuc/ PTEN-null-spheres (GSC23; Supplementary Fig. 17c,d,g). These histological features were observed after re-expression of DAXX and PTEN in animals bearing shDAXX/PTEN-null GBM-spheres (GSC23; Supplementary Fig. 17e,f,g). Our studies indicate that $D A X X$ suppression inhibits GBM-PDX tumour growth and extends overall survival specifically in mice engrafted with PTENdeficient GBM cells.
$D A X X$ expression is upregulated in gliomas. Having established that $D A X X$ disruption inhibits tumour growth and increases survival in GBM-PDX models, we next examined if DAXX gene expression was altered in different human gliomas. By using the REMBRANDT and TCGA databases we found a statistically significant $(P<0.0001)$ upregulation of $D A X X$ expression in GBMs, oligodendrogliomas and astrocytomas in comparison with normal brain (Fig. 6a). This was apparent in the classical, mesenchymal and proneural GBM subtypes (Fig. 6b). However, no significant changes in DAXX protein signal were observed within PTEN-positive and PTEN-negative adult GBM samples (Fig. 6c, Chi-square 0.7639, $P=0.6825, n=68$ ); but a significant anticorrelated expression (cor $=-0.298, P=0.001, n=166$ ) between DAXX and PTEN-WT was found in the GBM-TCGA database (Fig. 6d) and confirmed by immunohistochemistry in adult GBM tissues (Fig. 6e, cor $=-0.3721 P=0.0025, n=67$ ). A similar anticorrelated $D A X X / P T E N$ expression pattern was also observed in invasive breast carcinoma (BRCA, cor $=-0.325$, $P=1.26 \mathrm{e}-27, n=1,096)$ from the TCGA data set (Supplementary Fig. 18a); suggesting a more general cancer-related gene expression regulatory mechanism. No significant correlation was observed between ATRX/PTEN and HIRA/PTEN (Supplementary Fig. 18b,c), indicating a specific PTEN-DAXX regulatory mechanism.

We next investigated the biological consequence of DAXX upregulation in human GBMs. Gene set enrichment analysis showed that the three most overrepresented gene sets that positively correlated with DAXX expression in GBM patients were E2F targets, G2M checkpoint components and MYC targets (Fig. 6f,g); in concordance with our data showing that DAXX knockdown affects cell cycle progression, cellular proliferation (Supplementary Figs 4 and 5) and tumour growth (Fig. 5 and Supplementary Fig. 16). The prognostic impact of DAXX genetic alterations (mutations) in GBMs was also interrogated using the TCGA data set. We found that $1 \%$ of GBMs have DAXX alterations (missense mutations); however, a difference in overall survival rate compared with non-altered cases was not apparent (Supplementary Fig. 19a, $P$ value 0.544 ). The overall survival rate in patients with both DAXX and PTEN alterations was not significant ( $P$ value 0.976) in GBM cases (Supplementary Fig. 19b). Our analysis indicates that DAXX expression is upregulated in gliomas and inversely correlated with PTEN.

Finally, we analysed gene expression levels in the normal human brain using the Allen human brain database ${ }^{55}$. Here, it was determined that PTEN and H3F3B (H3.3) have similar expression profiles in comparison with CCND1, MYC, FOS and $B C L 2$ genes in the same brain structure regions (Supplementary Fig. 20). Particularly in the metencephalon (MET), the intensity of PTEN expression was 3-6 times higher than expression of the other analysed genes, using different probes. These analyses are consistent with our general model, proposing that PTEN controls gene expression through the regulation of H3.3 and DAXX.

\footnotetext{
Figure 4 | DAXX inhibition affects H3.3 genomic enrichment, gene expression and oncogenesis in PTEN-null/GBM cells. (a) Genomic distribution of H3.3 peaks in DAXX knockdown GBM cells. (b) Volcano plot of H3.3 differential binding (DiffBind) peaks between shControl (shLuc) and shDaxx PTEN-null/GBM samples by ChIP-seq $(P<0.05 \& \log 2$ (Fold Change) $>1$ ). (c) Gene ontology analysis of H3.3 differential binding genes in DAXX knockdown PTEN-deficient GBM-spheres. (d) Volcano plot of differentially expressed (DiffExp) gene between shLuc and shDaxx PTEN-null/GBM samples by RNA-seq $(P<0.05 \& \log 2$ (Fold Change) $>1$ ). (e) Heatmap of Top 100 DiffExp genes highlighting tumour suppressors genes and oncogenes according to the Cancer Gene Census in DAXX-kd compared with shControl (shLuc) cells ( $P$-value $<0.05$, log2(Fold change) $>1$ ). (f) Scatter plot of overlay genes between H3.3 DiffBind and DiffExp genes from ChIP- and RNA-seq, respectively. Red square, upregulated genes with H3.3 enrichment. Green square, downregulated genes with H3.3 enrichment $(P<0.05 \& \log 2$ (Fold Change) $>1$ ). Top 100 DiffExp genes are labelled with green dots in ChIP- and RNA-seq plots and scatter plot. $(\mathbf{g}, \mathbf{h})$ Quantification of the total number of spheres $(\mathbf{g})$ and sphere size $(\mathbf{h})$ in GBM-PDX lines transduced with shLuc or shDaxx $(n=3$ biological samples with six replicates each, NS: no significant differences, ${ }^{\star} P<0.05,{ }^{\star \star} P<0.001$ and ${ }^{\star \star *} P<0.0001$, one-way ANOVA). (i) Plots of sphere-forming frequencies using PTEN-NULL (GSC11 and GSC23) and PTEN-WT (GBM6 and TS576) GBM-PDX neurospheres, after stable knockdown of DAXX or shControl. The assay was performed by in vitro limiting dilution using a 0.95 confidence interval.
} 
a
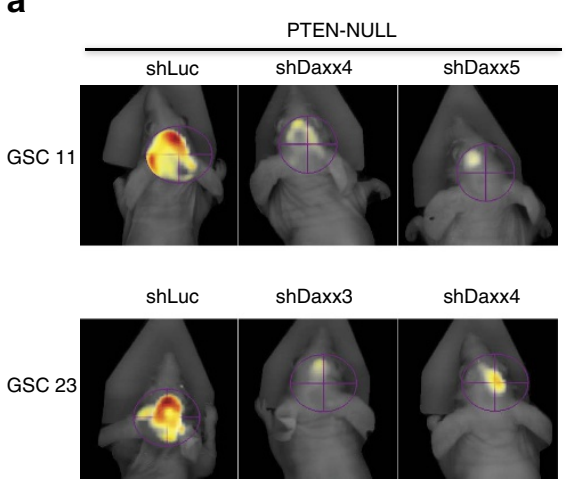

C

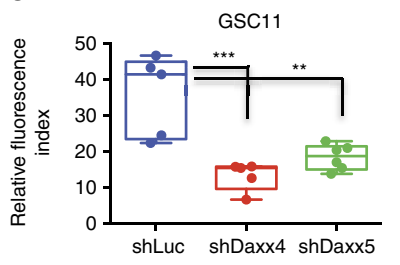

GSC23
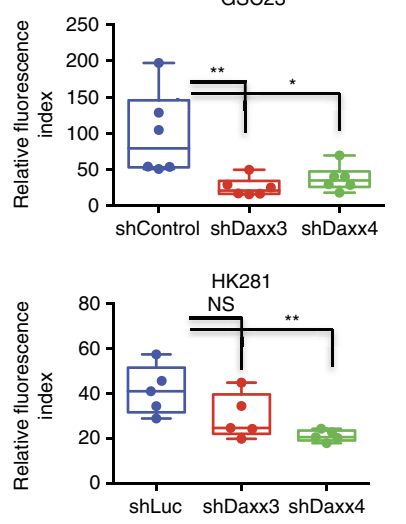

d
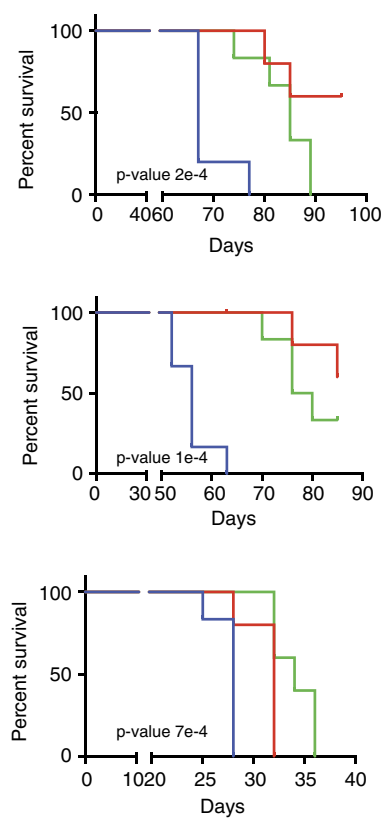

b
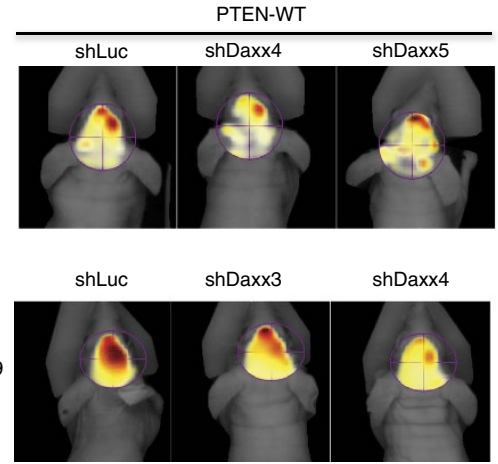

e

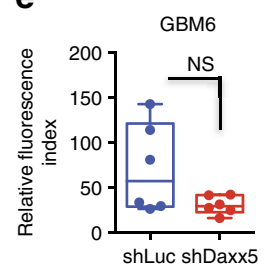

f
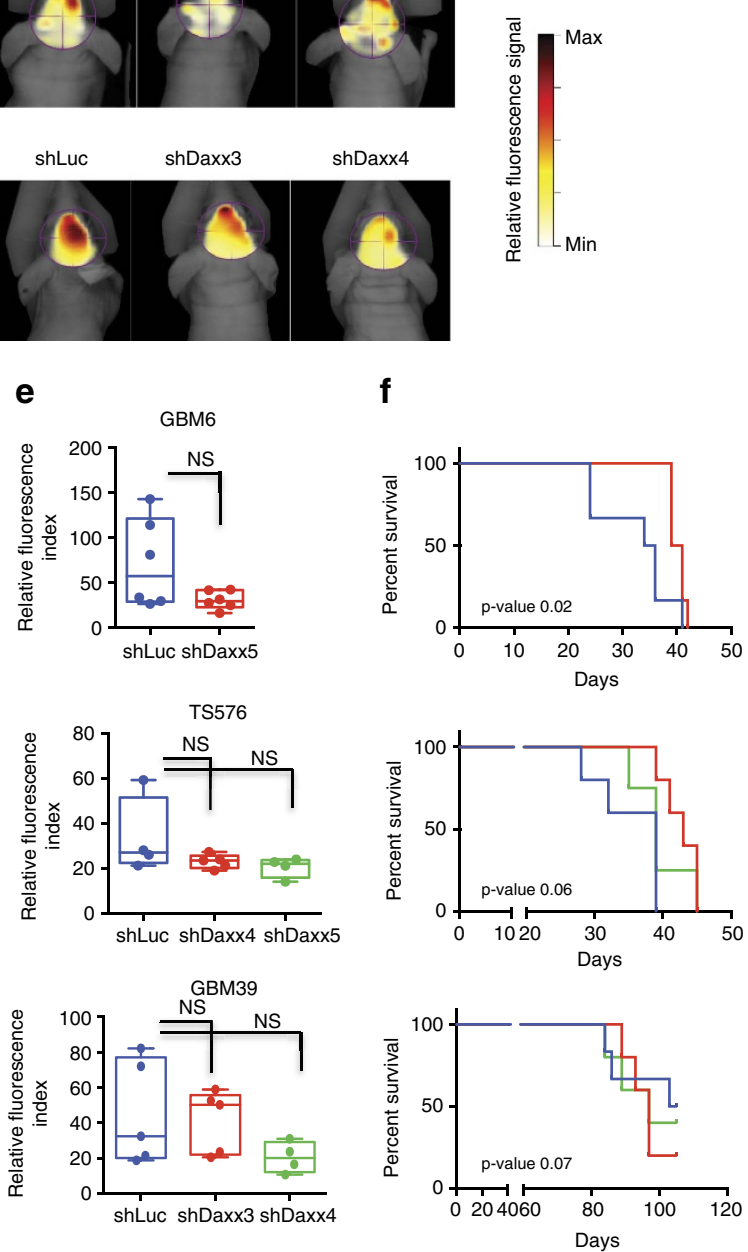

g
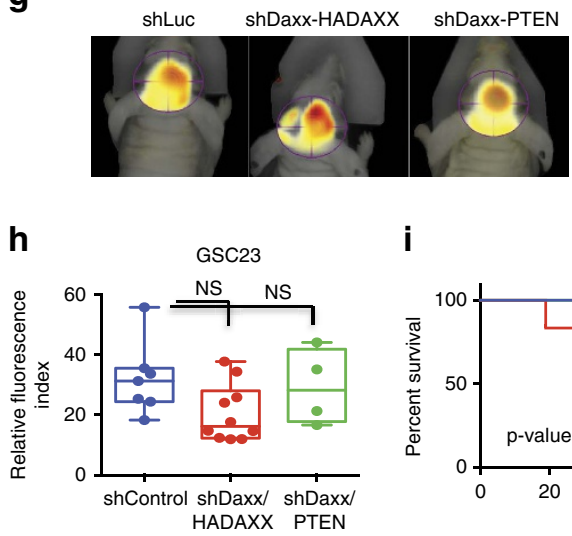

i
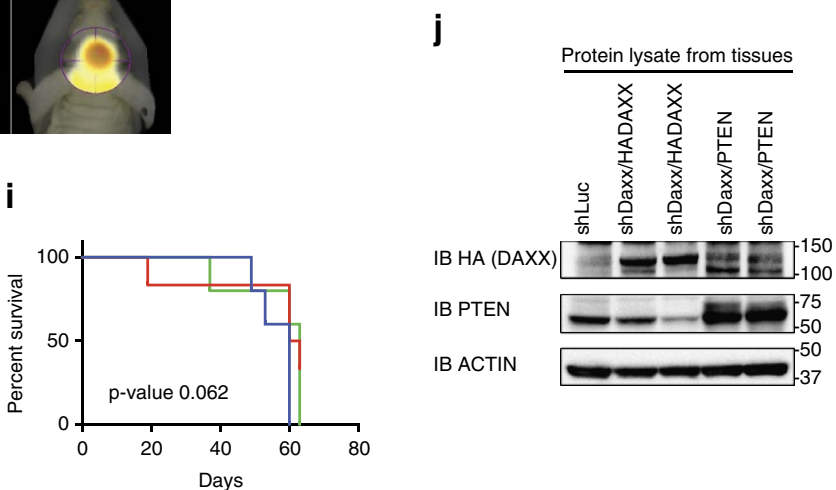

Figure 5 | DAXX disruption inhibits intracranial tumour growth and improves survival rate in PTEN-deficient GBM-PDX models. (a,b) Representative fluorescence molecular tomography (FMT) images of mice intracranially engrafted with PTEN-deficient (a) or PTEN-expressing (b) GBM-PDX neurospheres. Glioma spheres were co-transduced with lentivirus-encoded shRNAs targeting DAXX (shDaxx) or shRNA Control (shLuc), and a near infrared fluorescence protein. Relative fluorescence signal was monitored by FMT. (c,e) Relative fluorescence quantification of GSC11, GSC23 and HK281 PTEN-NULL (c), and GBM6, TS765 and GBM39 PTEN-WT (e) glioma-PDX xenografts analysed by FMT imaging $\left({ }^{\star} P<0.05,{ }^{\star \star} P<0.001\right.$ and ${ }^{\star \star \star} P<0.0001$, one-way ANOVA). (d,f) Kaplan-Meier survival curves of mice implanted with PTEN-deficient (d) or wild-type PTEN (f) GBM-PDXs expressing shDaxx or shControl. (g-j) In vivo rescue experiments in DAXX-kd/PTEN-deficient GSC23 engrafted mice after re-expression of HA-DAXX-shRNA-resistant (shDaxx/HADAXX) or PTEN-WT (shDaxx/PTEN). (g) Representative FMT images, (h) Relative fluorescence quantification, (i) Kaplan-Meier survival curves and (j) immunoblot analysis from tissues. PTEN antibody also detects endogenous mouse-Pten in shLuc and shDaxx samples. NS: no significant differences. 


\section{Discussion}

Our study suggests that PTEN is part of a chromatin complex with DAXX and H3.3, and negatively regulates genes involved in oncogenesis (Fig. 6h and Supplementary Fig. 21). Since DAXX recruits proteins like H3.3 to PML-nuclear bodies (PML-NBs) ${ }^{24,56}$, and PML-NBs have been shown to regulate PTEN $^{37}$, we propose that DAXX, H3.3, PML and PTEN may form a chromatin complex that regulates gene transcription. We suggest that in the absence of PTEN an unincorporated H3.3-chromatin fraction is recruited to PML-NBs in a DAXX-dependent manner ${ }^{56}$, leading to an increase in a DAXX-H3.3 soluble fraction. Upon DAXX inhibition, we speculate that $\mathrm{H} 3.3$ is liberated from PML-NBs and is hence restored for chromatin binding.

We propose a model that in PTEN-deficient tumour cells, DAXX removes H3.3 from chromatin (Fig. 6h, Supplementary Fig. 21a), probably by competing for chromatin binding, as has been reported by other groups ${ }^{45}$. Therefore, inhibition of DAXX restores $\mathrm{H} 3.3$ on the chromatin and inhibits oncogene expression (Fig. 6h, Supplementary Fig. 21b). The anti-tumorigenic effect mediated by DAXX inhibition does not work in PTEN-expressing cells because PTEN can also bind to $\mathrm{H} 3.3$ and we speculate that this attenuates H3.3 chromatin binding (Supplementary Fig. 21c).

Four tumour-related genes associated with neural stem cell proliferation and neuronal activity, and regulated by $\mathrm{PTEN}^{39-43}$ and DAXX ${ }^{35,38}$ were studied. Particularly, MYC and CCND1 have been reported to be upregulated after PTEN disruption in progenitor cells ${ }^{40,42}$, and associated with brain hyper-proliferation in a Pten knockout mouse model ${ }^{40}$. Here we demonstrate that PTEN impinges upon MYC and CCND1 expression at the transcriptional level by increasing the loading of a repressive DAXX-H3.3 complex on the chromatin. In contrast, MYC and CCND1 overexpression that occurs in the context of PTEN deficiency can be abrogated by $D A X X$ inhibition, which restores chromatin loading of repressive H3.3. Furthermore, genomic analysis in $D A X X$-knockdown/PTEN-deficient GBM samples display a genome-wide $\mathrm{H} 3.3$ distribution change (1,751 genes with $\mathrm{H} 3.3$ different binding signal) and upregulation of several tumour suppressor genes and downregulation of various oncogenes (1,403 genes with differential expression), including CCND1, MYC, FOS, SOX2 and OLIG2, compared with shControl/PTEN-deficient GBM samples. In concordance with the literature ${ }^{12,38,57}$, we show that H3.3 enrichment correlates with an upregulation and downregulation of genes involved in nervous system development and neuronal differentiation.

It is well documented that the $\mathrm{H} 3.3$ variant is regulated and incorporated in the chromatin by distinct proteins. Histone H3.3 is preferentially loaded at euchromatic regions by HIRA ${ }^{12,22}$ and at heterochromatic regions by $\mathrm{DAXX}^{23,24}$. Furthermore, the ATRX/DAXX complex is required for targeting H3.3 to telomeric chromatin $^{12}$. However, DAXX and ATRX have a distinct chromatin-binding profile, where DAXX preferentially binds to promoter regions ${ }^{58}$ and regulates $\mathrm{H} 3.3$ loading of immediate early genes after neuronal stimulation ${ }^{38}$. More recently, it was reported that MLL5 (Mixed Lineage Leukemia 5) represses H3.3 expression in adult GBMs allowing global reorganization of chromatin and self-renewal ${ }^{20}$. These data suggest that several histone chaperons and chromatin regulator proteins, including PTEN, can be involved in H3.3 deposition and its expression, and consequently chromatin regulation. In this study, we did not observe changes in the expression or localization of ATRX and HIRA in PTEN-deficient cells, no changes in DAXX-PTEN and DAXX-H3.3 association after ATRX overexpression, nor a correlated expression with PTEN-WT. However, we did detect a downregulation of expression of ATRX after DAXX inhibition, as has been reported previously ${ }^{12,23}$. Our results indicate that
DAXX disruption specifically affects GBM-PDX oncogenesis in PTEN-null models independently of ATRX.

$D A X X$ and ATRX mutations have also been correlated with an alternative lengthening of telomeres (ALT) phenotype in pancreatic neuroendocrine tumours ${ }^{59}$ and paediatric $\mathrm{GBMs}^{14}$; however, intact telomeres have been observed in PTEN-deficient cells $^{9}$, where chromosomal translocations and centromeres breakages are mainly affected. We reason that because genetic alterations of $D A X X$ are uncommon in adult GBMs, oncogene transcription and chromosomal instability may drive cellular transformation mediated by PTEN disruption and DAXX deregulation through nuclear functions.

Additionally, our studies show that DAXX inhibition in GBM-PDX neurospheres suppresses tumour growth and increases survival, specifically in a PTEN-deficient background, in part by negatively regulating the expression of oncogenes implicated in gliomagenesis. It has been shown that SOX2, MYC and OLIG2 are required to maintain proliferation in progenitor cells and they have been implicated in different types of cancer $28,51-53,60$. We suggest that downregulation of expression of these GBM-TFs can be associated with the PTEN-DAXX-H3.3 complex, since H3.3 is enriched near the transcription binding sites of these genes, as we observed from the genomic data and in concordance with the literature ${ }^{12}$, and DAXX disruption only affects their expression in PTEN-deficient cells.

In a therapeutic context, DAXX-H3.3 interaction can be disrupted in PTEN-null cells using staple peptides as reported by Kim and collegues ${ }^{61}$ for the disassociation of an EZH2-EDD complex in a leukaemia model; or by using small molecules which have been efficient at antagonizing chromatin associated proteins and their interactions with other proteins ${ }^{62}$. Another strategy to target $D A X X$ is by inhibiting its expression at the transcriptional level which can be attempted by performing high-throughput gene expression modulation by small molecules (GEMS) screening of compounds ${ }^{28,63}$.

In summary, we propose that PTEN-DAXX-H3.3 is a chromatin complex that regulates gene transcription (Fig. $6 \mathrm{~h}$ and Supplementary Fig. 21). Our study nominates DAXX as a new therapeutic target to revert tumorigenesis caused by PTEN loss of function in GBMs. Additionally, a DAXX-inhibition strategy offers an opportunity for other PTEN-null tumours where MYC and CYCLIN-D1 are upregulated, including medulloblastomas, endometrial cancer, breast cancer and melanoma ${ }^{8,46,47}$.

\section{Methods}

Reagents and antibodies. A detailed description of the antibodies, drugs and kits used in this work is presented in Supplementary Methods.

Cell culture and plasmids. MEFs and human glioma cell lines were cultured in DMEM plus $10 \%$ of fetal bovine serum (GIBCO/Life Technologies). Human glioblastoma patient-derived (GBM-PDX) spheres were maintained in DMEM/F12 1:1 medium with B27 supplement (GIBCO/Life Technologies) plus human recombinant EGF (20 $\mathrm{ng} \mathrm{ml}^{-1}$ ) and FGF $\left(10 \mathrm{ng} \mathrm{ml}^{-1}\right.$ ) (Stemcell Technologies). A detailed description of MEFs, glioma cells, GBM-PDX spheres and vectors is in Supplementary Methods.

In vitro pull-down assay. Fifty nanograms of human recombinant His-PTEN (ENZO Life Science) proteins were previously immobilized on $30 \mu \mathrm{l}$ of nickel-beads (GE Healthcare Life Sciences) and then incubated with $200 \mathrm{ng}$ of human recombinant Flag-DAXX protein (OriGene Technologies) for $2 \mathrm{~h}$ at $4^{\circ} \mathrm{C}$. Bound proteins were eluted and visualized by immunoblotting.

Immuno-precipitation experiments. For immune-precipitation analysis, whole-cell lysates or nuclear fractions were extracted following the manufacturer's instruction provided with the Universal Magnetic co-IP kit (54002, Active Motif). $300 \mu \mathrm{g}$ of proteins were immunoprecipitated using $2.0 \mu \mathrm{g}$ of antibody and $20 \mu \mathrm{l}$ of Dynabeads (10007D, life technologies). 
a

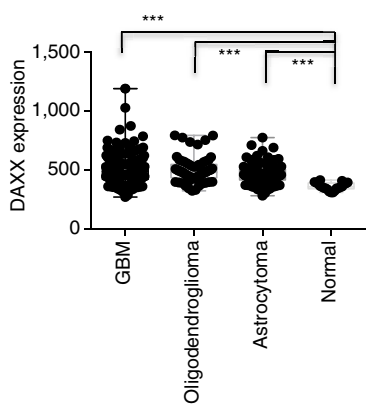

d

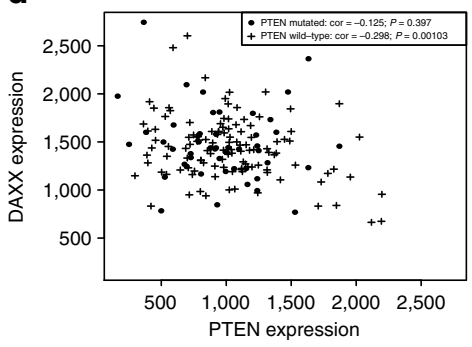

$\mathbf{f}$

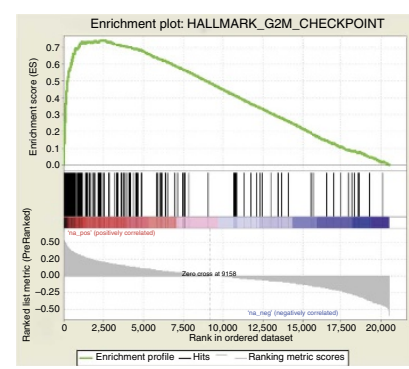

b

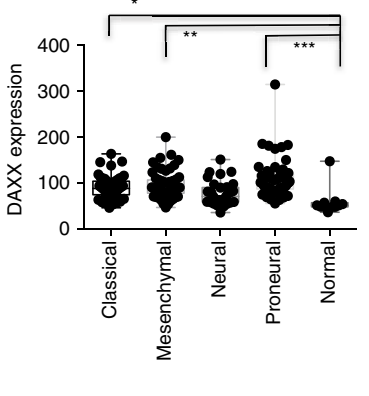

C

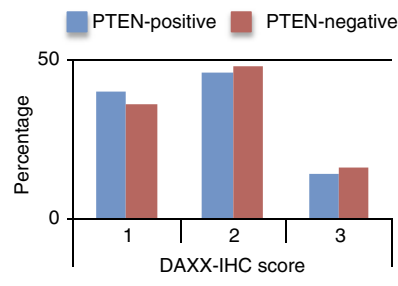

e

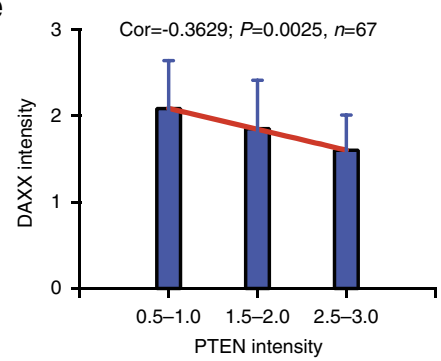

g

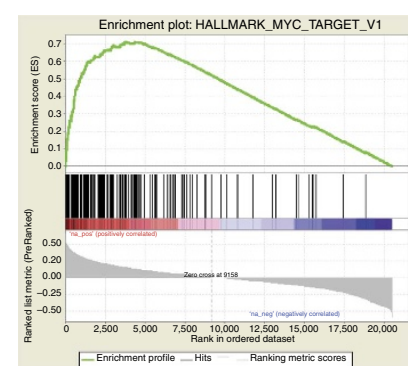

h

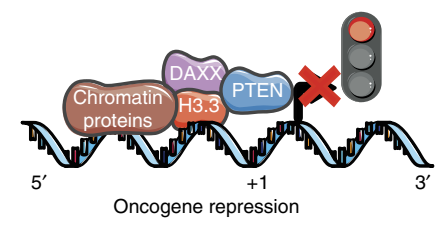

Oncogene repression

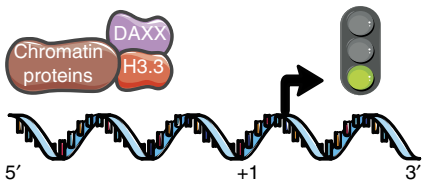

Oncogene transcription

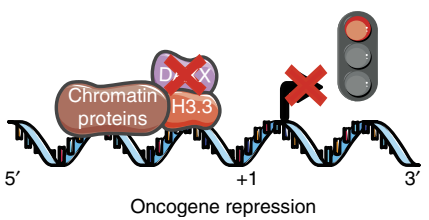

Figure 6 | DAXX expression is altered in GBMs. (a) REMBRANDT data analysis of DAXX expression in gliomas compared with normal brain. (b) TCGA data analysis of DAXX expression in GBM subtypes ( ${ }^{\star} P<0.05,{ }^{* \star} P<0.001$ and ${ }^{\star \star *} P<0.0001$, one-way ANOVA). (c) Immunohistochemistry analysis of DAXX expression signal in PTEN-positive and PTEN-negative adult GBM tissues (Chi-square 0.7639, $P=0.6825, n=68$ ). (d) Anti-correlated expression of DAXX and PTEN in GBMs from the TCGA database. (e) Immunohistochemistry quantification of DAXX and PTEN intensity signal from adult GBM tissues (cor $=-0.3721 P=0.0025, n=67)$. $(\mathbf{f}, \mathbf{g})$ Gene set enrichment analysis (GSEA) showing the most overrepresented gene sets among positively correlated: (f) G2M checkpoint and (g) MYC targets (enrichment score $=0.744$ and 0.713; FDR q-value $=0.000 ; F W E R P$ value $=0.000$ ). (h) Schematic diagram showing that in the presence of PTEN (top sketch) oncogene transcription is repressed by a PTEN-DAXX-H3.3 chromatin complex in association with other chromatin proteins. Conversely, upon loss of PTEN (middle sketch) oncogene transcription is activated. DAXX inhibition in the context of PTEN deficiency (bottom sketch) restores $\mathrm{H} 3.3$ on chromatin and hence represses oncogene transcription. 
RNA isolation and RT-QPCR. Total RNA was isolated with RNeasy kit (Qiagen), quantified and $1 \mu \mathrm{g}$ of RNA was reverse-transcribed with the Superscript II reagent (Invitrogen). Q-PCR was performed using SYBER Green mix (Bio-Rad). Primers are described in Supplementary Methods.

Lentivirus production and purification. Mission shRNA lentiviral particles (Sigma-Aldrich) were produced by co-transfection of shRNA pLKO-base lentivirus targeting Daxx or shRNA control vector, packaging gene vector pDELTA-8.9 and viral envelope vector pVSV-G in HEK293 cells with Lipofectamine 2000 (Life Technologies). A detailed description of protocol is in Supplementary Methods.

Chromatin immunoprecipitation. Chromatin was isolated from 2 million cells according to the manufacturer's recommended procedure in CHIP-IT kit (Active Motif 53040). Sheared chromatin (250 $\mu \mathrm{g})$ was immunoprecipitated using $5 \mu \mathrm{g}$ of ChIP quality antibody. ChIP-DNA was eluted $(200 \mu \mathrm{l}$ of elution buffer for ChIP-PCR or in $50 \mu \mathrm{l}$ for ChIP-seq), and $2 \mu \mathrm{l}$ were analysed by q-PCR using SYBER Green mix (Bio-Rad). Sheared chromatin $(25 \mu \mathrm{g})$ were used as Input-DNA. Primers were designed by Prime 3 and validated in the Genome Browser. Primers are described in Supplementary Methods.

Cellular fractionation. Subcellular protein fractions were extracted according to the manufacturer's instruction (Thermo Scientific) and $10 \mu \mathrm{g}$ of proteins were resolved by SDS-PAGE followed by immunoblotting.

Immunofluorescence microscopy. Cells were plated on poly-D-lysine-coated glass coverslips (Thermo Scientific), fixed with 10\% formalin (Sigma-Aldrih), blocked with $2 \%$ of BSA IgG-free (Jackson ImmunoResearch) and stained with primary antibodies overnight at $4{ }^{\circ} \mathrm{C}$. Secondary antibody was added for $1 \mathrm{~h}$ at room temperature. Coverslips were mounted on microscope glass slides using Fluro-Gel with DAPI (Electron Microscopy Science) followed by visualization using confocal microscopy (Leica SP5 confocal with resonant scanner). A detailed description of immunofluorescence acquisition and analysis is in Supplementary Methods.

Cell proliferation and cell cycle analysis. One thousand cells were grown in 96-well plates and $72 \mathrm{~h}$ later cell proliferation was analysed by WST1 (MK400, Clontech) or ATplite assay (6016941, Perkin-Elmer). For cell cycle progression, cells were fixed with $70 \%$ of ethanol overnight and stained with FxCycle PI/RNase staining solution (F10797, Life Technologies). DNA content was evaluated by flow cytometry (FACS).

Promoter reporter assay. A 1.5 and $1.0 \mathrm{~Kb}$ fragment upstream of the transcriptional start site of the human CCND1 and MYC genes, respectively, were cloned into the pLightSwicth vector (32001, Active Motif). A detailed description of the assay is in Supplementary Methods.

Sphere formation assay. Glioma stem cells were dissociated into single cells and 100 or 500 cells per well were plated in 96 -well plates. Total number of spheres and total number of cells, per well and per treatment, were determined after 14 days in culture. A detailed description of the analysis is in Supplementary Methods.

Flow cytometry analysis. Quantification of proteins associated to the fork replication complex was determined by flow cytometry. A detailed description of the method is in Supplementary Methods.

Differentiation of GBM-PDX spheres. GBM spheres were dissociated into single cells and plated on glass coverslips coated with poly-D-lysine in DMEM medium with $1 \%$ of FBS. Coverslips were processed for immunostaining 7 days after plating.

Lentivirus transduction of GBM-PDX spheres. Glioma spheres were co-transduced with purified lentivirus that encoded shRNAs anti-DAXX (shDAXX) or shRNA control (shLuc) at multiplicity of infection (MOI) 5 , and a near infrared fluorescence protein (IRFP720, PerkinElmer) MOI 5, for $96 \mathrm{~h}$.

Intracranial xenograft tumour model. Animal research experiments were conducted under the regulations of the UCSD Animal Care Program, protocol number S00192M. GBM-PDX spheres were collected and resuspended at 0.5 or $1.0 \times 10^{6}$ cells in $2 \mu \mathrm{l}$ of PBS per animal, then stereotactically injected into the striatum $(1.0 \mathrm{~mm}$ anteroposterior and 2.0 lateral from Bregma suture and $3 \mathrm{~mm}$ below the pial surface) of immunodeficient mice (Charles River laboratory).

Tumour size measurement and survival analysis. Animals were observed for neurological signs and the relative fluorescence signal of the xenografts were analysed by fluorescence molecular tomography (PerkinElmer) and quantified using TrueQuant 3.1 software (PerkinElmer). For survival analysis, animals were killed when they showed signs of distress and morbidity.

Densitometry quantification. Immunoblots were acquired with ChemiDocMP (Bio-Rad) and the intensity signal was quantified by densitometry analysis with Image Lab software.

Immunohistochemistry and tissue microarray. Slides were deparaffinized and rehydrated by washing steps of $3 \mathrm{~min}$ in xylene, xylene:ethanol 1:1, 100\% ethanol, $95 \%$ ethanol, $70 \%$ ethanol, $50 \%$ ethanol and water. After deparaffinization, sections were boiled in citrate buffer $(\mathrm{pH}$ 6.0) for $25 \mathrm{~min}$. Sections were then treated with $5 \%$ serum-blocking solution for $20 \mathrm{~min}$. A detailed description of the method is in Supplementary Methods.

In silico protein-protein interactions. New PTEN nuclear interacting complexes were simulated using the bioinformatics site Human Interactome $\mathrm{Map}^{31}$. A detailed description of the analysis is in Supplementary Methods.

Chromatin immunoprecipitation sequencing and analysis. Libraries were made with the Kapa Hyper Prep kit (Roche, KK8502), starting with 2.5 ng of IP DNA, ad amplified by 15 cycles of PCR amplification, according to the manufacturer's protocol. Libraries were quantified and sized by running them on an Agilent Tapestation, measuring concentration of QPCR (Kapa Universal Library Quantification kit, Roche, KK4824). The libraries were run on an Illumina 2500, v4 chemistry, using a single read 50 protocol. ChIP-Seq analysis is described in Supplementary Methods.

RNA sequencing and analysis. Total RNA was assessed for quality using an Agilent Tapestation, and all samples had RNA Integrity Numbers above 9.0. RNA libraries were generated using Illumina's TruSeq Stranded mRNA Sample Prep Kit (Illumina, RS-122-2101) following the manufacturer's instructions, modifying the shear time to $5 \mathrm{~min}$. RNA-Seq analysis is described in Supplementary Methods.

TCGA, REMBRANDT and Allen Brain analysis. Gene expression, genetic alterations and survival rate analysis from TCGA, REMBRANDT and Allen Human Brain Atlas are described in Supplementary Methods.

Statistical analysis. Data sets were analysed by unpaired $t$-test or multiple comparisons one-way ANOVA or two-way ANOVA according to the experiment using GraphPad Prism software. ${ }^{*} P<0.05,{ }^{* *} P<0.001$ and ${ }^{* * *} P<0.0001$. Kaplan-Meier curves and comparison of survival were analysed using Long-rank (Mantel-Cox) test.

Data availability. Data generated during the study have been deposited in Sequence Read Archive (SRA) SRP090820.

\section{References}

1. Cancer Genome Atlas Research, N.. Comprehensive genomic characterization defines human glioblastoma genes and core pathways. Nature 455, 1061-1068 (2008).

2. Parsons, D. W. et al. An integrated genomic analysis of human glioblastoma multiforme. Science 321, 1807-1812 (2008).

3. Verhaak, R. G. et al. Integrated genomic analysis identifies clinically relevant subtypes of glioblastoma characterized by abnormalities in PDGFRA, IDH1, EGFR, and NF1. Cancer Cell 17, 98-110 (2010).

4. Cantley, L. C. The phosphoinositide 3-kinase pathway. Science 296, 1655-1657 (2002).

5. Zhang, L. et al. Microenvironment-induced PTEN loss by exosomal microRNA primes brain metastasis outgrowth. Nature 527, 100-104 (2015).

6. Jiang, Z. et al. Phosphatase and tensin homologue deficiency in glioblastoma confers resistance to radiation and temozolomide that is reversed by the protease inhibitor nelfinavir. Cancer Res. 67, 4467-4473 (2007).

7. Mellinghoff, I. K. et al. Molecular determinants of the response of glioblastomas to EGFR kinase inhibitors. N. Engl. J. Med. 353, 2012-2024 (2005).

8. Juric, D. et al. Convergent loss of PTEN leads to clinical resistance to a PI(3)Kalpha inhibitor. Nature 518, 240-244 (2015)

9. Shen, W. H. et al. Essential role for nuclear PTEN in maintaining chromosomal integrity. Cell 128, 157-170 (2007).

10. Freeman, D. J. et al. PTEN tumor suppressor regulates p53 protein levels and activity through phosphatase-dependent and -independent mechanisms. Cancer Cell 3, 117-130 (2003).

11. Okumura, K., Zhao, M., Depinho, R. A., Furnari, F. B. \& Cavenee, W. K. Cellular transformation by the MSP58 oncogene is inhibited by its physical interaction with the PTEN tumor suppressor. Proc. Natl Acad. Sci. USA 102, 2703-2706 (2005). 
12. Goldberg, A. D. et al. Distinct factors control histone variant H3.3 localization at specific genomic regions. Cell 140, 678-691 (2010).

13. Wong, L. H. et al. Histone $\mathrm{H} 3.3$ incorporation provides a unique and functionally essential telomeric chromatin in embryonic stem cells. Genome Res. 19, 404-414 (2009).

14. Schwartzentruber, J. et al. Driver mutations in histone H3.3 and chromatin remodelling genes in paediatric glioblastoma. Nature 482, 226-231 (2012).

15. Wu, G. et al. Somatic histone $\mathrm{H} 3$ alterations in pediatric diffuse intrinsic pontine gliomas and non-brainstem glioblastomas. Nat. Genet. 44, 251-253 (2012).

16. Lewis, P. W. et al. Inhibition of PRC2 activity by a gain-of-function $\mathrm{H} 3$ mutation found in pediatric glioblastoma. Science 340, 857-861 (2013).

17. Bender, S. et al. Reduced H3K27me3 and DNA hypomethylation are major drivers of gene expression in $\mathrm{K} 27 \mathrm{M}$ mutant pediatric high-grade gliomas. Cancer Cell 24, 660-672 (2013).

18. Sturm, D. et al. Hotspot mutations in H3F3A and IDH1 define distinct epigenetic and biological subgroups of glioblastoma. Cancer Cell 22, 425-437 (2012).

19. Bjerke, L. et al. Histone H3.3. mutations drive pediatric glioblastoma through upregulation of MYCN. Cancer Discov. 3, 512-519 (2013).

20. Gallo, M. et al. MLL5 orchestrates a cancer self-renewal state by repressing the histone variant H3.3 and globally reorganizing chromatin. Cancer Cell 28, 715-729 (2015)

21. Burgess, R. J. \& Zhang, Z. Histone chaperones in nucleosome assembly and human disease. Nat. Struct. Mol. Biol. 20, 14-22 (2013).

22. Ray-Gallet, D. et al. Dynamics of histone $\mathrm{H} 3$ deposition in vivo reveal a nucleosome gap-filling mechanism for H3.3 to maintain chromatin integrity. Mol. Cell 44, 928-941 (2011).

23. Lewis, P. W., Elsaesser, S. J., Noh, K. M., Stadler, S. C. \& Allis, C. D. Daxx is an H3.3-specific histone chaperone and cooperates with ATRX in replicationindependent chromatin assembly at telomeres. Proc. Natl Acad. Sci. USA 107, 14075-14080 (2010).

24. Drane, P., Ouararhni, K., Depaux, A., Shuaib, M. \& Hamiche, A. The death-associated protein DAXX is a novel histone chaperone involved in the replication-independent deposition of H3.3. Genes Dev. 24, 1253-1265 (2010).

25. Jiao, Y. et al. DAXX/ATRX, MEN1, and mTOR pathway genes are frequently altered in pancreatic neuroendocrine tumors. Science 331, 1199-1203 (2011).

26. Cancer Genome Atlas Research, N. et al. Comprehensive, integrative genomic analysis of diffuse lower-grade gliomas. N. Engl. J. Med. 372, 2481-2498 (2015)

27. Johnson, B. E. et al. Mutational analysis reveals the origin and therapy-driven evolution of recurrent glioma. Science 343, 189-193 (2014).

28. Kreso, A. et al. Self-renewal as a therapeutic target in human colorectal cancer. Nat. Med. 20, 29-36 (2014)

29. Hashizume, R. et al. Pharmacologic inhibition of histone demethylation as a therapy for pediatric brainstem glioma. Nat. Med. 20, 1394-1396 (2014)

30. Funato, K., Major, T., Lewis, P. W., Allis, C. D. \& Tabar, V. Use of human embryonic stem cells to model pediatric gliomas with $\mathrm{H} 3.3 \mathrm{~K} 27 \mathrm{M}$ histone mutation. Science 346, 1529-1533 (2014).

31. Rhodes, D. R. et al. Probabilistic model of the human protein-protein interaction network. Nat. Biotechnol. 23, 951-959 (2005).

32. Zhao, L. Y. et al. Negative regulation of p53 functions by Daxx and the involvement of MDM2. J. Biol. Chem. 279, 50566-50579 (2004).

33. Lin, D. Y. \& Shih, H. M. Essential role of the 58-kDa microspherule protein in the modulation of Daxx-dependent transcriptional repression as revealed by nucleolar sequestration. J. Biol. Chem. 277, 25446-25456 (2002).

34. Zhong, S. et al. Promyelocytic leukemia protein (PML) and Daxx participate in a novel nuclear pathway for apoptosis. J. Exp. Med. 191, 631-640 (2000).

35. Puto, L. A. \& Reed, J. C. Daxx represses RelB target promoters via DNA methyltransferase recruitment and DNA hypermethylation. Genes Dev. 22 998-1010 (2008).

36. Wethkamp, N. \& Klempnauer, K. H. Daxx is a transcriptional repressor of CCAAT/enhancer-binding protein beta. J. Biol. Chem. 284, 28783-28794 (2009).

37. Song, M. S. et al. The deubiquitinylation and localization of PTEN are regulated by a HAUSP-PML network. Nature 455, 813-817 (2008).

38. Michod, D. et al. Calcium-dependent dephosphorylation of the histone chaperone DAXX regulates H3.3 loading and transcription upon neuronal activation. Neuron 74, 122-135 (2012).

39. Zheng, H. et al. p53 and Pten control neural and glioma stem/progenitor cell renewal and differentiation. Nature 455, 1129-1133 (2008).
40. Groszer, M. et al. Negative regulation of neural stem/progenitor cell proliferation by the Pten tumor suppressor gene in vivo. Science 294, 2186-2189 (2001).

41. Groszer, M. et al. PTEN negatively regulates neural stem cell self-renewal by modulating G0-G1 cell cycle entry. Proc. Natl Acad. Sci. USA 103, 111-116 (2006).

42. He, X. C. et al. PTEN-deficient intestinal stem cells initiate intestinal polyposis Nat. Genet. 39, 189-198 (2007).

43. Koul, D. et al. PTEN down regulates AP-1 and targets c-fos in human glioma cells via PI3-kinase/Akt pathway. Mol. Cell Biochem. 300, 77-87 (2007).

44. Liu, C. P. et al. Structure of the variant histone H3.3-H4 heterodimer in complex with its chaperone DAXX. Nat. Struct. Mol. Biol. 19, 1287-1292 (2012).

45. Elsasser, S. J. et al. DAXX envelops a histone H3.3-H4 dimer for H3.3-specific recognition. Nature 491, 560-565 (2012).

46. Musgrove, E. A., Caldon, C. E., Barraclough, J., Stone, A. \& Sutherland, R. L. Cyclin D as a therapeutic target in cancer. Nat. Rev. Cancer 11, 558-572 (2011)

47. Swartling, F. J. et al. Distinct neural stem cell populations give rise to disparate brain tumors in response to N-MYC. Cancer Cell 21, 601-613 (2012).

48. Zhou, B. B. et al. Tumour-initiating cells: challenges and opportunities for anticancer drug discovery. Nat. Rev. Drug Discov. 8, 806-823 (2009).

49. Sanai, N., Alvarez-Buylla, A. \& Berger, M. S. Neural stem cells and the origin of gliomas. N. Engl. J. Med. 353, 811-822 (2005).

50. Puto, L. A., Brognard, J. \& Hunter, T. Transcriptional repressor DAXX promotes prostate cancer tumorigenicity via suppression of autophagy. J. Biol. Chem. 290, 15406-15420 (2015).

51. Ligon, K. L. et al. Olig2-regulated lineage-restricted pathway controls replication competence in neural stem cells and malignant glioma. Neuron 53, 503-517 (2007)

52. Suva, M. L. et al. Reconstructing and reprogramming the tumor-propagating potential of glioblastoma stem-like cells. Cell 157, 580-594 (2014).

53. Suva, M. L., Riggi, N. \& Bernstein, B. E. Epigenetic reprogramming in cancer. Science 339, 1567-1570 (2013).

54. He, J., Kang, X., Yin, Y., Chao, K. S. \& Shen, W. H. PTEN regulates DNA replication progression and stalled fork recovery. Nat. Commun. 6, 7620 (2015).

55. Hawrylycz, M. J. et al. An anatomically comprehensive atlas of the adult human brain transcriptome. Nature 489, 391-399 (2012).

56. Delbarre, E., Ivanauskiene, K., Kuntziger, T. \& Collas, P. DAXX-dependent supply of soluble (H3.3-H4) dimers to PML bodies pending deposition into chromatin. Genome Res. 23, 440-451 (2013).

57. Maze, I. et al. Critical role of histone turnover in neuronal transcription and plasticity. Neuron 87, 77-94 (2015).

58. He, Q. et al. The Daxx/Atrx complex protects tandem repetitive elements during DNA hypomethylation by promoting H3K9 trimethylation. Cell Stem Cell 17, 273-286 (2015).

59. Heaphy, C. M. et al. Altered telomeres in tumors with ATRX and DAXX mutations. Science 333, 425 (2011).

60. Boumahdi, S. et al. SOX2 controls tumour initiation and cancer stem-cell functions in squamous-cell carcinoma. Nature 511, 246-250 (2014).

61. Kim, W. et al. Targeted disruption of the EZH2-EED complex inhibits EZH2-dependent cancer. Nat. Chem. Biol. 9, 643-650 (2013).

62. Grebien, F. et al. Pharmacological targeting of the Wdr5-MLL interaction in C/EBPalpha N-terminal leukemia. Nat. Chem. Biol. 11, 571-578 (2015).

63. Bhattacharyya, A., Trotta, C. R. \& Peltz, S. W. Mining the GEMS-a novel platform technology targeting post-transcriptional control mechanisms. Drug Discov. Today 12, 553-560 (2007).

\section{Acknowledgements}

We thank Philip Leder for Daxx MEFs; David James, Frederick Lang, Paul Mischel, Cameron Brennan and Harley Kornblum for GBM-PDX neurospheres, and Daiqing Liao for the pcDNA3.1-Daxx construct. Thanks to Jennifer Santini and Taylor Horwood for confocal microscopy support and the UCSD Neuroscience microscopy shared facility grant P30 NS047101. Aris Tsirigos and Adriana Heguy, NYU School of Medicine, Cancer Center grant P30CA016087 and Laura and Isaac Perlmutter Cancer Center. Kristen Jepsen, IGM Genomics Center-UCSD grant P30DK063491. We also thank ABTA, AACR and CSHL for their financial support to J.A.B. This work was supported by an award from the American Brain Tumor Society (to J.A.B.), grant support from the Defeat GBM Research Collaborative, a subsidiary of National Brain Tumor Society (to W.K.C., F.B.F.), and R01-NS080939 and the James S. McDonnell Foundation (to F.B.F). 


\section{Author contributions}

J.A.B., J.M, A.B., M.F.C., C.Z., and N.M.J. performed and analysed the experiments; J.A.B., M.A., H.M. and S.S. performed IHC assays and analyses; J.A.B, S.K. and A.K.-J. performed ChIP-seq analysis; J.A.B., M.A., and K.A.F. performed RNA-seq and TCGA bioinformatics analyses; J.A.B., W.K.C. and F.B.F designed the experiments and wrote the manuscript.

\section{Additional information}

Supplementary Information accompanies this paper at http://www.nature.com/ naturecommunications

Competing interests: The authors declare no competing financial interests.

Reprints and permission information is available online at http://npg.nature.com/ reprintsandpermissions/
How to cite this article: Benitez, J. A et al. PTEN regulates glioblastoma oncogenesis through chromatin-associated complexes of DAXX and histone H3.3. Nat. Commun. 8, 15223 doi: $10.1038 /$ ncomms15223 (2017).

Publisher's note: Springer Nature remains neutral with regard to jurisdictional claims in published maps and institutional affiliations.

(c) (i) This work is licensed under a Creative Commons Attribution 4.0 International License. The images or other third party material in this article are included in the article's Creative Commons license, unless indicated otherwise in the credit line; if the material is not included under the Creative Commons license, users will need to obtain permission from the license holder to reproduce the material To view a copy of this license, visit http://creativecommons.org/licenses/by/4.0/

(C) The Author(s) 2017 


\section{Publisher Correction: PTEN regulates glioblastoma oncogenesis through chromatin-associated complexes of DAXX and histone H3.3}

Jorge A. Benitez, Jianhui Ma, Matteo D'Antonio, Antonia Boyer, Maria Fernanda Camargo, Ciro Zanca, Stephen Kelly, Alireza Khodadadi-Jamayran, Nathan M. Jameson, Michael Andersen, Hrvoje Miletic, Shahram Saberi, Kelly A. Frazer, Webster K. Cavenee \& Frank B. Furnari

Nature Communications 8:15223 doi: 10.1038/ncomms15223 (2017); Published 12 May 2017; Updated 25 May 2018

The originally published version of this Article contained an error in Fig. 1. In panel d, the uppermost western blot was inadvertently inverted during typesetting of the figure. This has now been corrected in both the PDF and HTML versions of the Article.

\footnotetext{
(c) (i) This work is licensed under a Creative Commons Attribution 4.0 International License. The images or other third party material in this article are included in the article's Creative Commons license, unless indicated otherwise in the credit line; if the material is not included under the Creative Commons license, users will need to obtain permission from the license holder to reproduce the material. To view a copy of this license, visit http://creativecommons.org/licenses/by/4.0/

(C) The Author(s) 2018
} 